\title{
Cumulative effects of incorrect use of pesticides can lead to catastrophic outbreaks of pests
}

\author{
Xia Wang ${ }^{\mathrm{a}}$, Zihui $\mathrm{Xu}^{\mathrm{a}}$, Sanyi Tang ${ }^{\mathrm{a}, *}$, Robert A. Cheke ${ }^{\mathrm{b}}$ \\ ${ }^{a}$ School of Mathematics and Information Science, Shaanxi Normal University, Xi'an, \\ 710119, P.R. China \\ ${ }^{b}$ Natural Resources Institute, University of Greenwich at Medway, Central Avenue, \\ Chatham Maritime, Chatham, Kent, ME4 4TB, UK
}

\begin{abstract}
Modeling external perturbations such as chemical control within each generation of discrete populations is challenging. Based on a method proposed in the literature, we have extended a discrete single species model with multiple instantaneous pesticide applications within each generation, and then discuss the existence and stability of the unique positive equilibrium. Further, the effects of the timing of pesticide applications and the instantaneous killing rate on the equilibrium were investigated in more detail and we obtained some interesting results, including a paradox and the cumulative effects of the incorrect use of pesticides on pest outbreaks, . In order to show the occurrences of the paradox and of hormesis, several special models have been extended and studied. Further, the biological implications of the main results regarding successful pest control are discussed. All of the results obtained confirm that the cumulative effects of incorrect use of pesticides may result in more severe pest outbreaks and thus, in order to avoid a paradox in pest control, control strategies need to be designed with care, including decisions on the timing and number of pesticide applications in relation to the effectiveness of the pesticide being used.
\end{abstract}

Keywords: Non-overlapping generation; Multiple pesticide applications; Paradox; Cumulative effect; Beverton-Holt model; Ricker model.

*Corresponding author. Tel: +862985310232

Email addresses: xiawang@snnu.edu.cn (Xia Wang), sytang@snnu.edu.cn, sanyitang219@hotmail.com (Sanyi Tang) 


\section{Introduction}

The continuous Lotka-Volterra model first proposed by Volterra in 1926 which has been widely used in fishery resources management and pest control [1, 2, 45, 46, 26, 47], and Lotka-Volterra's famous principle reveals that an intervention in the Lotka-Volterra model that kills or removes both pest and natural enemies in proportion to their population densities has the effect of increasing average pest populations. This conclusion indicates that control tactics must be designed carefully when an integrated pest management (IPM) strategy including chemical and biological controls is employed in practice.

Much theoretical and practical research has addressed how to mitigate and control pest outbreaks $[9,10,11,12,13,14,15,16,17]$. Although the application of pesticides is the most commonly used pest control method and it can be very effective at the beginning of a campaign, in the later periods it often results in an outbreak of the pest, a phenomenon known as pest resurgence $[18,19,20,21,22]$. Note that there are two possible reasons for a resurgence: one is the emergence of a pesticide-resistant strain of the pest, and the other is a decrease in its enemy population as a result of the pesticide applications [23]. Furthermore, low doses of a pesticide can enhance a pests fecundity, while high doses can reduce the pest population density [23, 24], a phenomenon called hormesis a term which describes generally favorable biological responses to low exposures to toxins and other stressors [23].

To understand more details about the mechanism of resurgence and hormesis for successful pesticide applications in pest control, mathematical models play an important role in determining the effectiveness of pesticides and the optimal timing of pesticide applications [44, 7, 8]. As already mentioned, continuous models including Lotka-Volterra models and pest and natural enemy systems with IPM strategies [44, 43, 26, 47] have revealed that human actions have significant effects on pest resurgence and hormesis.

However, most pests have non-overlapping generations and control action$\mathrm{s}$ are applied within each generation, thus the question is how to formulate and involve external perturbations into discrete population models. In order to address this question, some interesting modeling methods have been proposed recently $[5,4,25]$, and the main results indicate that the timing of an external perturbation and its strength could significantly affect on the dynamical behavior. In particular, harvesting/thinning can cause an eventual increase of the targets population at the equilibrium when incorrect human 
actions have been implemented, which shows that single pesticide applications within each pest generation may result in a pest outbreak.

Therefore, multiple pesticide applications should be applied once the first pesticide application has been unable to control the pest population successfully, then the most interesting questions are: (1) how to model the multiple pesticide applications within each generation and analyze the dynamical behavior? (2) how do successive applications of pesticide affect each other? (3) can the cumulative effects of incorrect use of pesticides lead to catastrophic outbreaks of pests or not? To answer these questions, we first develop a discrete single species model with multiple pesticide applications within each population generation, and then the existence and stability of the unique positive equilibrium was investigated. Furthermore, the effects of the timing of pesticide applications and the instantaneous killing rate on the equilibrium have been investigated in more detail, and some interesting results, including on the cumulative effects of incorrect use of pesticides on pest outbreaks, have been obtained. In order to show the occurrences of a paradox and hormesis, several special models discussed in the literature $[5,4,25]$ have been extended and studied, with some new and interesting results contrasting with those obtained in the cited references. Finally, the biological implications of the main results for successful pest control are discussed, which can help us to design successful pest control strategies when the pest population has non-overlapping generations.

\section{Multiple control strategies applied within each pest generation}

Recently, the following discrete single species population model $[5,4,25]$

$$
x_{n+1}=\lambda(1-q) x_{n}\left\{\theta g\left(x_{n}\right)+(1-\theta) g\left((1-q) x_{n}\right)\right\}
$$

with one control event within generation $n$ and $n+1$ has been proposed and investigated. Here $x_{n} \in[0,+\infty)$ is the population size at generation $n \in N$, and $N$ denotes the integer set. The smooth function $g(x)(x \geq 0)$ shows the intra-specific density effect on the reproduction rate which satisfies $0<g(x) \leq 1$. The positive parameter $\lambda$ represents the intrinsic growth rate, and then $\lambda g(x)$ with $\lambda>1$ means the per capita reproductive rate with respect to the intra-specific density [25]. In model (2.1) the control measure is applied instantaneously within the generation $[n, n+1]$, which indicates that there exists a positive constant $\theta$ with $0 \leq \theta \leq 1$ such that the control 
measure including spraying pesticide is applied at time point $n+\theta$, and a proportion $q(0 \leq q<1)$ of the pest population has been killed at the moment of $n+\theta$. Thus after the time point $n+\theta$, the density dependent growth of the species population depends on $(1-q) x_{n}[25]$.

In particular, if $\theta=0$ then model (2.1) can be changed to the following impulsive difference equation

$$
\begin{cases}x_{n+1}=\lambda x_{n} g\left(x_{n}\right), & n=0,1,2, \cdots \\ x_{n^{+}}=(1-q) x_{n}, & n=0,1,2, \cdots\end{cases}
$$

which indicates that the chemical control tactics have been applied at the beginning of each generation or parasitism season. For more details of impulsive difference equations, please see the reference [43]. If $\theta=1$ then model (2.1) can be changed into the following impulsive difference equation

$$
\left\{\begin{array}{l}
x_{n+1}=\lambda x_{n} g\left(x_{n}\right), \quad n=0,1,2, \cdots \\
x_{(n+1)^{+}}=(1-q) x_{n+1}, \quad n=0,1,2, \cdots,
\end{array}\right.
$$

which shows that the chemical control tactics have been applied at the end of each generation or parasitism season. For convenience, we assume that the function $g(x)$ affected by the density is twice continuously differentiable, and for any $x>0$ the $g(x)$ satisfies $g(0)=1, \lim _{x \rightarrow \infty} g(x)=0$ and $g^{\prime}(x)=$ $\frac{d g(x)}{d x}<0$.

In reality, the chemical control tactics could be applied several times within each pest generation, dependent on the pest outbreak frequency and/or limited resources. Therefore, the main purpose of this work is to introduce multiple chemical control tactics into model (2.1) and to investigate the effects of these multiple control measures on the interesting dynamics obtained in reference [25]. To do this, we assume that pesticides have been applied $m$ times within generation $[n, n+1]$ satisfying $n \leq n+\theta_{1} \leq n+\theta_{2} \leq \cdots \leq$ $n+\theta_{m} \leq n+1$, and at each time point $n+\theta_{i}$, a proportion $q_{i}\left(0 \leq q_{i}<1\right)$ of the population has been killed, and then $1-q_{i}$ denotes the survival rate at time point $n+\theta_{i}$. Therefore, we have the following model

$$
x_{n+1}=\lambda \prod_{i=1}^{m}\left(1-q_{i}\right) x_{n}\left[\sum_{i=0}^{m}\left(\Delta \theta_{i} g\left(\prod_{j=0}^{i}\left(1-q_{j}\right) x_{n}\right)\right)\right],
$$

where $\Delta \theta_{i}=\theta_{i+1}-\theta_{i}$ for $i=0,1,2, \cdots, m$, and without loss of the generality we assume that $\theta_{0}=0, \theta_{m+1}=1$ and $q_{0}=0$. 


\section{Generalized analyses}

In this section, we first analyze the existence and stability of the equilibrium of model (2.2), and then discuss the effects of the timing of applications $\theta_{i}$ and the instantaneous killing rate $q_{i}$ on the equilibrium.

\subsection{Existence and stability of non-trivial equilibrium}

The equilibrium population size $x^{*}$ satisfies the following equation

$$
1=\lambda \prod_{i=1}^{m}\left(1-q_{i}\right)\left[\sum_{i=0}^{m}\left(\Delta \theta_{i} g\left(\prod_{j=0}^{i}\left(1-q_{j}\right) x^{*}\right)\right)\right] .
$$

According to $\lambda>1$ and the properties of the function $g(x)$, we can easily see that

$$
\lambda \prod_{i=1}^{m}\left(1-q_{i}\right)>1
$$

is the necessary condition for the existence of positive equilibrium $x^{*}$. Consequently, if $\lambda \prod_{i=1}^{m}\left(1-q_{i}\right) \leq 1$, i.e. $\prod_{i=1}^{m}\left(1-q_{i}\right) \geq 1-\frac{1}{\lambda}$, then Eq.(3.1) does not have any positive root $x^{*}$, and further the population size is gradually reducing in the $[n, n+1]$ generation and becomes extinct eventually.

Define a new function $r(x)$ according to Eq.(3.1) as follows

$$
r(x)=\left[\sum_{i=0}^{m}\left(\Delta \theta_{i} g\left(\prod_{j=0}^{i}\left(1-q_{j}\right) x\right)\right)\right]-\frac{1}{\lambda \prod_{i=1}^{m}\left(1-q_{i}\right)},
$$

and taking the derivative of $r(x)$ with respect to $x$, yields

$$
r^{\prime}(x)=\left[\sum_{i=0}^{m}\left(\Delta \theta_{i} g^{\prime}\left(\prod_{j=0}^{i}\left(1-q_{j}\right) x\right)\left(\prod_{j=0}^{i}\left(1-q_{j}\right)\right)\right)\right] .
$$

It follows from $g^{\prime}(x)<0$ that $r^{\prime}(x)<0$, indicating that the function $r(x)$ is a monotonically decreasing function with respect to $x>0$.

Based on the basic properties of the function $g(x)$, it follows from the assumption $\lambda \prod_{i=1}^{m}\left(1-q_{i}\right)>1$ that we have

$$
r(0)=1-\frac{1}{\lambda \prod_{i=1}^{m}\left(1-q_{i}\right)}>0,
$$




$$
\lim _{x \rightarrow+\infty} r(x)=-\frac{1}{\lambda \prod_{i=1}^{m}\left(1-q_{i}\right)}<0,
$$

which means that there is a unique $x=x^{*} \in(0,+\infty)$ such that $r\left(x^{*}\right)=0$. That is to say, Eq.(3.1) has a unique positive root provided that $\lambda \prod_{i=1}^{m}(1-$ $\left.q_{i}\right)>1$.

Thus, the equilibrium $x=x^{*}>0$ uniquely exists if and only if $\lambda \prod_{i=1}^{m}(1-$ $\left.q_{i}\right)>1$. Further, in the case of $x^{*}>0$, we can have the following result with the standard local stability analysis, i.e. the equilibrium $x=x^{*}>0$ is asymptotically stable if

$$
\left|\frac{d}{d x}\left\{x\left[\sum_{i=0}^{m}\left(\Delta \theta_{i} g\left(\prod_{j=0}^{i}\left(1-q_{j}\right) x\right)\right)\right]\right\}\right|_{x=x^{*}}<1 .
$$

If the inequality is reversed, the equilibrium $x=x^{*}>0$ is unstable.

3.2. The effects of timing of application pesticide and killing rate on the equilibrium

Although we cannot solve the equilibrium $x^{*}$ analytically, $x^{*}$ strictly depends on the timings of the applications of the pesticide $\theta_{i}$ and the killing rates $q_{i}$ for $i=1,2, \cdots, m$. Therefore, in this subsection, we address how the equilibrium $x^{*}$ varies as the parameters $\theta_{i}$ and $q_{i}$ change, which are quite important for proper pesticide applications.

Firstly, from Eq.(3.1) taking the derivation of $x^{*}$ with respect to $q_{k}$, yields

$$
\frac{\partial x^{*}}{\partial q_{k}}=-\frac{F_{q_{k}}}{F_{x^{*}}}, k=1,2, \cdots m
$$

with

$$
\begin{gathered}
F_{x^{*}}=\lambda \prod_{i=1}^{m}\left(1-q_{i}\right)\left\{\sum_{i=0}^{m}\left(\Delta \theta_{i} g^{\prime}\left(\prod_{j=0}^{i}\left(1-q_{j}\right) x^{*}\right) \prod_{j=0}^{i}\left(1-q_{j}\right)\right)\right\} \\
F_{q_{k}}=-\lambda \prod_{i=1, i \neq k}^{m}\left(1-q_{i}\right) \sum_{i=0}^{m}\left(\Delta \theta_{i} g\left(\prod_{j=0}^{i}\left(1-q_{j}\right) x^{*}\right)\right)+A
\end{gathered}
$$

and

$$
A=\lambda \prod_{i=1}^{m}\left(1-q_{i}\right)\left\{\sum_{i=k}^{m}\left(\Delta \theta_{i} g^{\prime}\left(\prod_{j=0}^{i}\left(1-q_{j}\right) x^{*}\right) \prod_{j=0, j \neq k}^{i}\left(1-q_{j}\right)\left(-x^{*}\right)\right)\right\} .
$$


Therefore, we have

$$
\begin{aligned}
\frac{\partial x^{*}}{\partial q_{k}} & =\frac{C+\left(1-q_{k}\right)\left\{\sum_{i=k}^{m}\left(\Delta \theta_{i} g^{\prime}\left(\prod_{j=0}^{i}\left(1-q_{j}\right) x^{*}\right) \prod_{j=0, j \neq k}^{i}\left(1-q_{j}\right) x^{*}\right)\right\}}{\left(1-q_{k}\right)\left\{\sum_{i=0}^{m}\left(\Delta \theta_{i} g^{\prime}\left(\prod_{j=0}^{i}\left(1-q_{j}\right) x^{*}\right) \prod_{j=0}^{i}\left(1-q_{j}\right)\right)\right\}} \\
& =\frac{C+\left\{\sum_{i=k}^{m}\left(\Delta \theta_{i} g^{\prime}\left(\prod_{j=0}^{i}\left(1-q_{j}\right) x^{*}\right) \prod_{j=0}^{i}\left(1-q_{j}\right) x^{*}\right)\right\}}{\left(1-q_{k}\right)\left\{\sum_{i=0}^{m}\left(\Delta \theta_{i} g^{\prime}\left(\prod_{j=0}^{i}\left(1-q_{j}\right) x^{*}\right) \prod_{j=0}^{i}\left(1-q_{j}\right)\right)\right\}}
\end{aligned}
$$

where $C=\sum_{i=0}^{m}\left(\Delta \theta_{i} g\left(\prod_{j=0}^{i}\left(1-q_{j}\right) x^{*}\right)\right)$. due to

$$
\begin{gathered}
\frac{\partial}{\partial q_{k}}\left[\left(1-q_{k}\right) \sum_{i=0}^{m}\left(\Delta \theta_{i} g\left(\prod_{j=0}^{i}\left(1-q_{j}\right) x^{*}\right)\right)\right] \\
=C+\left\{\sum_{i=k}^{m}\left(\Delta \theta_{i} g^{\prime}\left(\prod_{j=0}^{i}\left(1-q_{j}\right) x^{*}\right) \prod_{j=0}^{i}\left(1-q_{j}\right) x^{*}\right)\right\} .
\end{gathered}
$$

Thus, we have

$$
\frac{\partial x^{*}}{\partial q_{k}}=\frac{-\frac{\partial}{\partial q_{k}}\left[\left(1-q_{k}\right) \sum_{i=0}^{m}\left(\Delta \theta_{i} g\left(\prod_{j=0}^{i}\left(1-q_{j}\right) x^{*}\right)\right)\right]}{\left(1-q_{k}\right)\left[\sum_{i=0}^{m}\left(\Delta \theta_{i} \prod_{j=0}^{i}\left(1-q_{j}\right) g^{\prime}\left(\prod_{j=0}^{i}\left(1-q_{j}\right) x^{*}\right)\right)\right]} .
$$

It follows from $g^{\prime}(x)<0$ that we have

$$
\left(1-q_{k}\right)\left[\sum_{i=0}^{m}\left(\Delta \theta_{i} \prod_{j=0}^{i}\left(1-q_{j}\right) g^{\prime}\left(\prod_{j=0}^{i}\left(1-q_{j}\right) x^{*}\right)\right)\right]<0 .
$$

Therefore, we conclude that increasing the instantaneous killing rate $q_{k}$ can result in increasing the equilibrium population size if and only if the following condition

$$
\frac{\partial}{\partial q_{k}}\left(1-q_{k}\right)\left[\sum_{i=0}^{m}\left(\Delta \theta_{i} g\left(\prod_{j=0}^{i}\left(1-q_{j}\right) x^{*}\right)\right)\right]>0
$$

holds true.

The condition shown in Eq.(3.5) indicates that, under certain conditions, the larger the control tactic is the more severe is the outbreak. If so, we call this an occurrence of the emergence of the paradox. That is to say, the 
reduction of the density of the population by the $k$-th pesticide application within each generation can cause the increasing of the population size if the control tactics have not been properly applied. On the other hand, if $\frac{\partial}{\partial q_{k}}\left(1-q_{k}\right)\left[\sum_{i=0}^{m}\left(\Delta \theta_{i} g\left(\prod_{j=0}^{i}\left(1-q_{j}\right) x^{*}\right)\right)\right] \leq 0$ for any $x^{*}>0$, then the equilibrium population size $x^{*}$ is monotonically decreasing with the increasing of $q_{k}(k=1,2, \cdots, m)$, so that the paradox will never occur in this case.

The effects of $\theta_{k}$ on the equilibrium population size $x^{*}$ can be calculated as follows:

$$
\frac{\partial x^{*}}{\partial \theta_{k}}=-\frac{F_{\theta_{k}}}{F_{x^{*}}}, k=1,2, \cdots m
$$

with

$$
\begin{aligned}
F_{\theta_{k}} & =\lambda \prod_{i=1}^{m}\left(1-q_{i}\right) \frac{d}{d_{\theta_{k}}}\left[\left(\theta_{k}-\theta_{k-1}\right) g\left(\prod_{j=0}^{k-1}\left(1-q_{j}\right) x^{*}\right)+\left(\theta_{k+1}-\theta_{k}\right) g\left(\prod_{j=0}^{k}\left(1-q_{j}\right) x^{*}\right)\right] \\
& =\lambda \prod_{i=1}^{m}\left(1-q_{i}\right)\left[g\left(\prod_{j=0}^{k}\left(1-q_{j}\right) x^{*}\right)-g\left(\prod_{j=0}^{k-1}\left(1-q_{j}\right) x^{*}\right)\right]
\end{aligned}
$$

and

$$
F_{x^{*}}=\lambda \prod_{i=1}^{m}\left(1-q_{i}\right)\left\{\sum_{i=0}^{m}\left(\Delta \theta_{i} g^{\prime}\left(\prod_{j=0}^{i}\left(1-q_{j}\right) x^{*}\right) \prod_{j=0}^{i}\left(1-q_{j}\right)\right)\right\} .
$$

Thus, we have

$$
\frac{\partial x^{*}}{\partial \theta_{k}}=-\frac{g\left(\prod_{j=0}^{k-1}\left(1-q_{j}\right) x^{*}\right)-g\left(\prod_{j=0}^{k}\left(1-q_{j}\right) x^{*}\right)}{\sum_{i=0}^{m}\left[\Delta \theta_{i}\left(\prod_{j=0}^{i}\left(1-q_{j}\right)\right) g^{\prime}\left(\prod_{j=0}^{i}\left(1-q_{j}\right) x^{*}\right)\right]} .
$$

Then we can see that $\frac{\partial x^{*}}{\partial \theta_{k}}<0$ due to $g^{\prime}(x)<0$ and $\left(\prod_{j=0}^{k-1}\left(1-q_{j}\right) x^{*}\right)>$ $\left(\prod_{j=0}^{k}\left(1-q_{j}\right) x^{*}\right)$. That is to say, the paradox does not occur at all if we only consider the $x^{*}$ as a function of the timing of pesticide applications. However, it does affect the value of equilibrium $x^{*}$ when we consider the timing of pesticide applications and the instantaneous killing rate together, i.e. $x^{*}$ is a function of both variables $\left(q_{k}, \theta_{k}\right)$, which will be discussed in more detail below.

Specially, if $m=1$, then we can have the following equation based on Eq.(3.4)

$$
\frac{\partial x^{*}}{\partial q}=\frac{1}{(1-q) \Psi}\left\{\theta g\left(x^{*}\right)+(1-\theta) \frac{\partial}{\partial x^{*}}\left[x^{*} g\left((1-q) x^{*}\right)\right]\right\},
$$


where $\Psi=\theta g^{\prime}\left(x^{*}\right)+(1-\theta)(1-q) g^{\prime}\left((1-q) x^{*}\right)$. Moreover, $\frac{\partial x^{*}}{\partial q}>0$ only if

$$
\theta g\left(x^{*}\right)+(1-\theta) \frac{\partial}{\partial x^{*}}\left[x^{*} g\left((1-q) x^{*}\right)\right]<0
$$

holds true. As a result of that $\theta g\left(x^{*}\right)>0$ and $(1-\theta)>0$, we can easily find that when $m=1$ the paradox occurs only if $\frac{\partial}{\partial x^{*}}\left[x^{*} g\left((1-q) x^{*}\right)\right]<0$, which have been investigated in [25].

It is easy to see that the inequality shown in Eq.(3.6) does not hold for $\theta=1$. That is to say, when we apply the pesticide at the later period of the specific season $[n, n+1]$, the equilibrium population size will be decreased as the killing rate increases, and then the paradox does not occur. This confirms that only a sufficiently small $\theta$ can ensure the occurrence of the paradox.

The successive applications of pesticides can cause different effects on the outbreak patterns and result in complex dynamics. In particular, the effects of the instantaneous killing rate $q_{k}$ on the value of $x^{*}$ can be summarised as in the following main results.

Theorem 1: If we consider the equilibrium $x^{*}$ as a function of $q_{k}$, then we have

$$
\frac{\partial x^{*}}{\partial q_{k}} \leq 0 \Rightarrow \frac{\partial x^{*}}{\partial q_{k+1}}<0, \quad \frac{\partial x^{*}}{\partial q_{k}}>0 \Rightarrow \frac{\partial x^{*}}{\partial q_{k-1}}>0 .
$$

Proof: It follows from Eq.(3.4) that we have the following three equations

1. $\frac{\partial x^{*}}{\partial q_{k-1}}=\frac{\left[\sum_{i=0}^{m} \Delta \theta_{i} g\left(\prod_{j=0}^{i}\left(1-q_{j}\right) x^{*}\right)\right]+\left[\sum_{i=k-1}^{m} \Delta \theta_{i} g^{\prime}\left(\prod_{j=0}^{i}\left(1-q_{j}\right) x^{*}\right) \prod_{j=0}^{i}\left(1-q_{j}\right) x^{*}\right]}{\left(1-q_{k-1}\right)\left[\sum_{i=0}^{m} \Delta \theta_{i} g^{\prime}\left(\prod_{j=0}^{i}\left(1-q_{j}\right) x^{*}\right) \prod_{j=0}^{i}\left(1-q_{j}\right) x^{*}\right]}$,

2. $\frac{\partial x^{*}}{\partial q_{k}}=\frac{\left[\sum_{i=0}^{m} \Delta \theta_{i} g\left(\prod_{j=0}^{i}\left(1-q_{j}\right) x^{*}\right)\right]+\left[\sum_{i=k}^{m} \Delta \theta_{i} g^{\prime}\left(\prod_{j=0}^{i}\left(1-q_{j}\right) x^{*}\right) \prod_{j=0}^{i}\left(1-q_{j}\right) x^{*}\right]}{\left(1-q_{k}\right)\left[\sum_{i=0}^{m} \Delta \theta_{i} g^{\prime}\left(\prod_{j=0}^{i}\left(1-q_{j}\right) x^{*}\right) \prod_{j=0}^{i}\left(1-q_{j}\right) x^{*}\right]}$,

3. $\frac{\partial x^{*}}{\partial q_{k+1}}=\frac{\left[\sum_{i=0}^{m} \Delta \theta_{i} g\left(\prod_{j=0}^{i}\left(1-q_{j}\right) x^{*}\right)\right]+\left[\sum_{i=k+1}^{m} \Delta \theta_{i} g^{\prime}\left(\prod_{j=0}^{i}\left(1-q_{j}\right) x^{*}\right) \prod_{j=0}^{i}\left(1-q_{j}\right) x^{*}\right]}{\left(1-q_{k+1}\right)\left[\sum_{i=0}^{m} \Delta \theta_{i} g^{\prime}\left(\prod_{j=0}^{i}\left(1-q_{j}\right) x^{*}\right) \prod_{j=0}^{i}\left(1-q_{j}\right) x^{*}\right]}$.

As a result of $g^{\prime}(x)<0$ we have

$$
\begin{cases}\left(1-q_{k-1}\right)\left[\sum_{i=0}^{m} \Delta \theta_{i} g^{\prime}\left(\prod_{j=0}^{i}\left(1-q_{j}\right) x^{*}\right) \prod_{j=0}^{i}\left(1-q_{j}\right) x^{*}\right] & <0, \\ \left(1-q_{k}\right)\left[\sum_{i=0}^{m} \Delta \theta_{i} g^{\prime}\left(\prod_{j=0}^{i}\left(1-q_{j}\right) x^{*}\right) \prod_{j=0}^{i}\left(1-q_{j}\right) x^{*}\right] & <0, \\ \left(1-q_{k+1}\right)\left[\sum_{i=0}^{m} \Delta \theta_{i} g^{\prime}\left(\prod_{j=0}^{i}\left(1-q_{j}\right) x^{*}\right) \prod_{j=0}^{i}\left(1-q_{j}\right) x^{*}\right] & <0 .\end{cases}
$$

Let $\Omega=\sum_{i=0}^{m} \Delta \theta_{i} g\left(\prod_{j=0}^{i}\left(1-q_{j}\right) x^{*}\right)$ and it is easy to know $\Omega>0$. Further, if $\frac{\partial x^{*}}{\partial q_{k}} \leq 0$, then the following inequality

$$
\Omega+\left[\sum_{i=k}^{m} \Delta \theta_{i} g^{\prime}\left(\prod_{j=0}^{i}\left(1-q_{j}\right) x^{*}\right) \prod_{j=0}^{i}\left(1-q_{j}\right) x^{*}\right] \geq 0
$$


holds true. The left side of Eq.(3.7) can be rewritten as follows

$$
\begin{aligned}
& \Omega+\left[\Delta \theta_{k} g^{\prime}\left(\prod_{j=0}^{k}\left(1-q_{j}\right) x^{*}\right)\left(\prod_{j=0}^{k}\left(1-q_{j}\right) x^{*}\right)\right] \\
+ & {\left[\sum_{i=k+1}^{m} \Delta \theta_{i} g^{\prime}\left(\prod_{j=0}^{i}\left(1-q_{j}\right) x^{*}\right) \prod_{j=0}^{i}\left(1-q_{j}\right) x^{*}\right] . }
\end{aligned}
$$

It follows from Eq.(3.7) and $\left[\Delta \theta_{k} g^{\prime}\left(\prod_{j=0}^{k}\left(1-q_{j}\right) x^{*}\right)\left(\prod_{j=0}^{k}\left(1-q_{j}\right) x^{*}\right)\right]<$ 0 that

$$
\left\{\Omega+\left[\sum_{i=k+1}^{m} \Delta \theta_{i} g^{\prime}\left(\prod_{j=0}^{i}\left(1-q_{j}\right) x^{*}\right) \prod_{j=0}^{i}\left(1-q_{j}\right) x^{*}\right]\right\}>0 .
$$

All these confirm that $\frac{\partial x^{*}}{\partial q_{k}} \leq 0$ indicates $\frac{\partial x^{*}}{\partial q_{k+1}}<0$.

If $\frac{\partial x^{*}}{\partial q_{k}}>0$, i.e. Eq. $(3.7)<0$, then it follows from

$$
\left[\Delta \theta_{k-1} g^{\prime}\left(\prod_{j=0}^{k-1}\left(1-q_{j}\right) x^{*}\right)\left(\prod_{j=0}^{k-1}\left(1-q_{j}\right) x^{*}\right)\right]<0
$$

that we can get

$$
\begin{aligned}
& \Omega+\left[\Delta \theta_{k-1} g^{\prime}\left(\prod_{j=0}^{k-1}\left(1-q_{j}\right) x^{*}\right)\left(\prod_{j=0}^{k-1}\left(1-q_{j}\right) x^{*}\right)\right] \\
& +\left[\sum_{i=k}^{m} \Delta \theta_{i} g^{\prime}\left(\prod_{j=0}^{i}\left(1-q_{j}\right) x^{*}\right) \prod_{j=0}^{i}\left(1-q_{j}\right) x^{*}\right]<0 .
\end{aligned}
$$

Further, the left side of Eq.(3.8) can be rewritten as follows:

$$
\Omega+\left[\sum_{i=k-1}^{m} \Delta \theta_{i} g^{\prime}\left(\prod_{j=0}^{i}\left(1-q_{j}\right) x^{*}\right) \prod_{j=0}^{i}\left(1-q_{j}\right) x^{*}\right] .
$$

This shows that $\frac{\partial x^{*}}{\partial q_{k}}>0$ indicates $\frac{\partial x^{*}}{\partial q_{k-1}}>0$. This completes the proof.

The main results shown in Theorem 1 reveal that if the $k$-th application of pesticide cannot cause the paradox, then the $(k+1)$-th application of pesticide also cannot result in the paradox, as shown in Fig.1. However if the $k$-th application of pesticide can cause the paradox, then the $(k-1)$-th application of pesticide may also cause the paradox under condition (3.5). All these results show that the chemical control tactics should be carefully designed before they are applied in practice, otherwise they will result in more severe outbreaks in terms of the density of the pest population.

In order to compare the main results obtained in [25] for single pesticide applications within each generation with the results for the proposed model in the present work, we employ the following three specific functions of $g(x)$ which include a Beverton-Holt type function, exponential function and power function to address how the number of times that the pesticide is applied per generation and the instantaneous killing rate affect the emergence of the paradox, and consequently on the control of the pest population. 


\section{Application and special cases}

\subsection{Beverton-Holt type function}

In this subsection, the following function of $g(x)$

$$
g(x)=\frac{1}{1+b x}
$$

has been chosen $[27,28,29]$, in which $b$ is a positive constant. Substituting Eq.(4.1) into model (2.2), yields the following Beverton-Holt model with multiple chemical controls within each generation:

$$
x_{n+1}=\lambda \prod_{i=1}^{m}\left(1-q_{i}\right) x_{n}\left\{\sum_{i=0}^{m}\left[\Delta \theta_{i}\left(1+b \prod_{j=0}^{i}\left(1-q_{j}\right) x_{n}\right)^{-1}\right]\right\} .
$$

The equilibrium population size $x^{*}$ satisfies the following equation:

$$
1=\lambda \prod_{i=1}^{m}\left(1-q_{i}\right)\left\{\sum_{i=0}^{m}\left[\Delta \theta_{i}\left(1+b \prod_{j=0}^{i}\left(1-q_{j}\right) x^{*}\right)^{-1}\right]\right\} .
$$

For this case, the function of $r(x)$ becomes as follows:

$$
r(x)=\left\{\sum_{i=0}^{m}\left[\Delta \theta_{i}\left(1+b \prod_{j=0}^{i}\left(1-q_{j}\right) x\right)^{-1}\right]\right\}-\frac{1}{\lambda \prod_{i=1}^{m}\left(1-q_{i}\right)},
$$

which satisfies the following conditions:

1. $r^{\prime}(x)<0$ for all $x>0$,

2. $r(0)=1-\frac{1}{\lambda \prod_{i=1}^{m}\left(1-q_{i}\right)}>0$ provided $\lambda \prod_{i=1}^{m}\left(1-q_{i}\right)>1$,

3. $\lim _{x \rightarrow \infty} r(x)=-\frac{1}{\lambda \prod_{i=1}^{m}\left(1-q_{i}\right)}<0$.

It follows from the main results obtain in Section 3.1 that model (4.2) exists a unique equilibrium $x=x^{*}$ provided that $\lambda \prod_{i=1}^{m}\left(1-q_{i}\right)>1$.

Further, we define the function $f\left(x^{*}\right)$ as follows:

$$
f\left(x^{*}\right)=\lambda \prod_{i=1}^{m}\left(1-q_{i}\right) x^{*}\left\{\sum_{i=0}^{m}\left[\Delta \theta_{i}\left(1+b \prod_{j=0}^{i}\left(1-q_{j}\right) x^{*}\right)^{-1}\right]\right\}
$$


and by simple calculation we have

$$
f^{\prime}\left(x^{*}\right)=\lambda \prod_{i=1}^{m}\left(1-q_{i}\right)\left\{\sum_{i=0}^{m}\left[\Delta \theta_{i}\left(1+b \prod_{j=0}^{i}\left(1-q_{j}\right) x^{*}\right)^{-2}\right]\right\} .
$$

Obviously $f^{\prime}\left(x^{*}\right)>0$, and according to $\left(1+b \prod_{j=0}^{i}\left(1-q_{j}\right) x^{*}\right)^{-2}<$ $\left(1+b \prod_{j=0}^{i}\left(1-q_{j}\right) x^{*}\right)^{-1},(3.2)$ and Eq.(4.3), we have $f^{\prime}\left(x^{*}\right)<1$. This indicates that $\left|f^{\prime}\left(x^{*}\right)\right|<1$ if the equilibrium $x^{*}$ exists, and consequently the equilibrium $x^{*}$ of model (4.2) is locally asymptotically stable.

Whether the paradox for model (4.2) may emerge or not with respect to the killing rate $q_{k}$ depends on condition (3.4), i.e. it depends on the sign of the following equation:

$$
\frac{\partial x^{*}}{\partial q_{k}}=-\frac{\sum_{i=k}^{m}\left(b x \cdot \Lambda \cdot \prod_{j=0}^{i}\left(1-q_{j}\right)\right)+\sum_{i=0}^{m}\left(\left(1+b x \cdot \prod_{j=0}^{i}\left(1-q_{j}\right)\right) \cdot \Lambda\right)}{\left(1-q_{k}\right)\left(\sum_{i=0}^{m}\left(b \cdot \Lambda \cdot \prod_{j=0}^{i}\left(1-q_{j}\right)\right)\right.}
$$

with $\Lambda=\frac{\Delta \theta_{i}}{\left(1+b x\left(\prod_{j=0}^{i}\left(1-q_{j}\right)\right)\right)^{2}}>0$. It is easy to see that $\frac{\partial x^{*}}{\partial q_{k}} \leq 0$, i.e. the paradox will never occur in model $(4.2)$ for any $q_{k}(k=1,2, \cdots, m)$ and $x^{*}>$ 0 . All these results confirm that if the pest population follows the BevertonHolt model, then successive control measures within the $n$-th generation can reduce the density of the pest population gradually.

In order to show in more detail how the timings of the pesticide applications and killing rates affect on equilibrium population size, we carried out numerical analysis in the rest of this subsection. In particular, we focus on how the multiple chemical control tactics affect the final size $x^{*}$. To do this, in Fig. 2 we fix $m=2$ and $q_{1}=q_{2}=q, \theta_{0}=0, \theta_{1}=\theta_{0}+\theta, \theta_{2}=\theta_{1}+\theta$. The main results shown in Fig.2 reveal that increasing the killing rate $q$ can result in decreasing the equilibrium of population size $x^{*}$, and the later the application (i.e. the larger $\theta$ ) is, the faster is the reducing effect, when $q$ is small.

For different killing rates and timings of pesticide applications, the results shown in Fig.3 indicate that no matter what the values of the $\theta_{1}$ and $\theta_{2}$ are, the equilibrium of population size $x^{*}$ will decrease with the increasing of $q_{1}$ and $q_{2}$. But the timings of pesticide applications are crucial in this case, i.e. with the increasing of $\theta_{1}$ and $\theta_{2}$ the equilibrium of population size $x^{*}$ reduces faster. All these results confirm again that the paradox will never occur if the pest population follows Beverton-Holt growth. 


\subsection{Ricker model or exponential function}

In this subsection, we choose another important function, i.e. the exponential function

$$
g(x)=e^{-\beta x}
$$

as a candidate to show the effects of the timing of pesticide applications and the killing rate on the pest outbreaks, and here the parameter $\beta$ is a positive constant. Note that the classical discrete Ricker model and its applications have been widely investigated [34, 33, 32, 31]. Substituting Eq.(4.4) into model (2.2), yields the following Ricker model with multiple chemical controls within each generation:

$$
x_{n+1}=\lambda \prod_{i=1}^{m}\left(1-q_{i}\right) x_{n}\left\{\sum_{i=0}^{m}\left[\Delta \theta_{i} e^{-\beta\left(\prod_{j=0}^{i}\left(1-q_{j}\right) x_{n}\right)}\right]\right\} .
$$

The equilibrium population size $x^{*}$ satisfies the following equation:

$$
1=\lambda \prod_{i=1}^{m}\left(1-q_{i}\right)\left\{\sum_{i=0}^{m}\left[\Delta \theta_{i} e^{-\beta\left(\prod_{j=0}^{i}\left(1-q_{j}\right) x^{*}\right)}\right]\right\} .
$$

For this case, the function of $r(x)$ becomes as follows:

$$
r(x)=\left\{\sum_{i=0}^{m}\left[\Delta \theta_{i} e^{-\beta\left(\prod_{j=0}^{i}\left(1-q_{j}\right) x\right)}\right]\right\}-\frac{1}{\lambda \prod_{i=1}^{m}\left(1-q_{i}\right)},
$$

and it is easy to check that

1. $r^{\prime}(x)<0$ for all $x>0$,

2. $r(0)=1-\frac{1}{\lambda \prod_{i=1}^{m}\left(1-q_{i}\right)}>0$ provided that $\lambda \prod_{i=1}^{m}\left(1-q_{i}\right)>1$,

3. $\lim _{x \rightarrow \infty} r(x)=-\frac{1}{\lambda \prod_{i=1}^{m}\left(1-q_{i}\right)}<0$,

which indicates that for model (4.5) there exists a unique equilibrium $x=x^{*}$ provided that $\lambda \prod_{i=1}^{m}\left(1-q_{i}\right)>1$.

Next we show the effects of the timings of pesticide applications and the killing rates on the stability of the equilibrium $x=x^{*}$ of Ricker model (4.5). To do this, it follows from

$$
f(x)=\lambda \prod_{i=1}^{m}\left(1-q_{i}\right) x\left\{\sum_{i=0}^{m}\left[\Delta \theta_{i} e^{-\beta\left(\prod_{j=0}^{i}\left(1-q_{j}\right) x\right)}\right]\right\}
$$


that we have

$$
\begin{aligned}
f^{\prime}\left(x^{*}\right)= & \lambda \prod_{i=1}^{m}\left(1-q_{i}\right)\left\{\sum_{i=0}^{m}\left[\Delta \theta_{i} e^{-\beta\left(\prod_{j=0}^{i}\left(1-q_{j}\right) x^{*}\right)}\right]\right. \\
& \left.-x^{*} \sum_{i=0}^{m}\left[\Delta \theta_{i}\left(\prod_{j=0}^{i}\left(1-q_{j}\right)\right) \beta e^{-\beta\left(\prod_{j=0}^{i}\left(1-q_{j}\right) x^{*}\right)}\right]\right\} \\
= & 1-\beta x^{*} \sum_{i=0}^{m}\left[\Delta \theta_{i}\left(\prod_{j=0}^{i}\left(1-q_{j}\right)\right) e^{-\beta\left(\prod_{j=0}^{i}\left(1-q_{j}\right) x^{*}\right)}\right] .
\end{aligned}
$$

Note that $\left|f^{\prime}\left(x^{*}\right)\right|<1$ indicates that the equilibrium $x^{*}$ is locally stable, i.e. we need

$$
0<\beta x^{*} \sum_{i=0}^{m}\left[\Delta \theta_{i}\left(\prod_{j=0}^{i}\left(1-q_{j}\right)\right) e^{-\beta\left(\prod_{j=0}^{i}\left(1-q_{j}\right) x^{*}\right)}\right]<2 .
$$

It is easy to see that

$$
0<\sum_{i=0}^{m}\left[\Delta \theta_{i}\left(\prod_{j=0}^{i}\left(1-q_{j}\right)\right) e^{-\beta\left(\prod_{j=0}^{i}\left(1-q_{j}\right) x^{*}\right)}\right]<\sum_{i=0}^{m}\left[\Delta \theta_{i}\right]=1,
$$

which indicates that if $0<\beta x^{*}<2$, then we have $\left|f^{\prime}\left(x^{*}\right)\right|<1$, i.e. the equilibrium $x^{*}$ is locally stable. Moreover, we note that the inequalities $0<\beta x^{*}<2$ ensure that the equilibrium of the classical Ricker model is locally stable. The effects of the timings of the pesticide applications and the killing rates on the stability of the equilibrium $x=x^{*}$ can be determined by the term

$$
\sum_{i=0}^{m}\left[\Delta \theta_{i}\left(\prod_{j=0}^{i}\left(1-q_{j}\right)\right) e^{-\beta\left(\prod_{j=0}^{i}\left(1-q_{j}\right) x^{*}\right)}\right],
$$

which is a function of both the timings of the pesticide applications $\theta_{i}$ and the killing rates $q_{i}$. All these results confirm that the application of multiple control tactics within each generation can enlarge the stable domain of the equilibrium.

What we want to address in the following is whether the paradox for Ricker model (4.5) may emerge or not with respect to the killing rate $q_{k}$ based 
on condition (3.4), i.e. the sign of $\frac{\partial x^{*}}{\partial q_{k}}$. Denote $\Phi_{i}=\Delta \theta_{i} e^{-\beta x^{*}\left(\prod_{j=0}^{i}\left(1-q_{j}\right)\right)}$, and we have

$$
\begin{gathered}
F_{x^{*}}=\lambda \prod_{i=1}^{m}\left(1-q_{i}\right)\left[\sum_{i=0}^{m} \Phi_{i}\left(-\beta\left(\prod_{j=0}^{i}\left(1-q_{j}\right)\right)\right)\right] \\
F_{q_{k}}=\lambda \prod_{i=1}^{m}\left(1-q_{i}\right)\left(\sum_{i=k}^{m} \Phi_{i}\left(\beta\left(\prod_{j=0, j \neq k}^{i}\left(1-q_{j}\right) x^{*}\right)\right)\right)-\lambda \prod_{i=1, i \neq k}^{m}\left(1-q_{i}\right)\left(\sum_{i=0}^{m} \Phi_{i}\right),
\end{gathered}
$$

which show that

$$
\begin{aligned}
\frac{\partial x^{*}}{\partial q_{k}} & =-\frac{F_{q_{k}}}{F_{x^{*}}}=\frac{\left(1-q_{k}\right)\left(\sum_{i=k}^{m} \Phi_{i} \cdot \beta \prod_{j=0, j \neq k}^{i}\left(1-q_{j}\right) x^{*}\right)-\left(\sum_{i=0}^{m} \Phi_{i}\right)}{\left(1-q_{k}\right)\left(\sum_{i=0}^{m} \Phi_{i} \cdot \beta \prod_{j=0}^{i}\left(1-q_{j}\right)\right)} \\
& =\frac{\left(\sum_{i=0}^{m} \Phi_{i}\right)-\left(\sum_{i=k}^{m} \Phi_{i} \cdot \beta \prod_{j=0}^{i}\left(1-q_{j}\right) x^{*}\right)}{\left(q_{k}-1\right)\left(\sum_{i=0}^{m} \Phi_{i} \cdot \beta \prod_{j=0}^{i}\left(1-q_{j}\right)\right)} \\
& =\frac{\left(\sum_{i=0}^{k-1} \Phi_{i}\right)+\left(\sum_{i=k}^{m} \Phi_{i}\right)-\left(\sum_{i=k}^{m} \Phi_{i} \cdot \beta \prod_{j=0}^{i}\left(1-q_{j}\right) x^{*}\right)}{\left(q_{k}-1\right)\left(\sum_{i=0}^{m} \Phi_{i} \cdot \beta \prod_{j=0}^{i}\left(1-q_{j}\right)\right)} .
\end{aligned}
$$

Therefore,

$$
\frac{\partial x^{*}}{\partial q_{k}}=\frac{\left[\sum_{i=0}^{k-1} \Phi_{i}\right]+\left[\sum_{i=k}^{m} \Phi_{i}\left(1-\beta x^{*}\left(\prod_{j=0}^{i}\left(1-q_{j}\right)\right)\right)\right]}{\beta\left(q_{k}-1\right) \sum_{i=0}^{m} \Phi_{i}\left(\prod_{j=0}^{i}\left(1-q_{j}\right)\right)} .
$$

Based on the condition of the emergence of the paradox, we can easily find that the sign of $\left[\sum_{i=0}^{k-1} \Phi_{i}\right]+\left[\sum_{i=k}^{m} \Phi_{i}\left(1-\beta x^{*}\left(\prod_{j=0}^{i}\left(1-q_{j}\right)\right)\right)\right]$ determines the existence of the paradox in the Ricker model (4.5). The problem is that we cannot obtain the concrete equilibrium $x^{*}$ from Eq.(4.6). So we carried out numerical analyses, as described in the rest of this subsection.

Similarly, for comparative reasons we consider one-time chemical control in Fig.(4)(a, c, e) with $m=1, \theta_{1}=\theta$ and $q_{1}=q$, two-times chemical controls in Fig.(4)(b, d, f) with $m=2, q_{1}=q_{2}=q, \Delta \theta_{i}=\theta_{i+1}-\theta_{i}(i=0,1,2), \theta_{0}=0$, $\theta_{1}=\theta_{0}+\theta, \theta_{2}=\theta_{1}+\theta$ and $\theta_{3}=1$. The effects of the killing rate $q$ and $\theta$ on the stable population level $x^{*}$ are shown in Fig.(4) by using bifurcation analyses.

It follows from the bifurcation diagrams shown in Fig.4 that the paradox occurs only for relatively small $\theta$ values, which means that increasing the killing rate will result in increasing the stable population level. Meanwhile, we note that there exists a threshold killing rate $q_{c}$ which monotonically decreases with increasing numbers of pesticide applications, such that the stable 
population size $x^{*}$ increases with the increasing of $q$, and consequently the paradox occurs. Moreover, the numbers of pesticide applications within each generation also play a key role for pest control, in particular for mitigating the paradox, as shown in Fig.4(e,f).

To show in more detail how the timing of pesticide applications and the killing rate affect the stable population size $x^{*}$, we fix the parameter values as those in Fig.5, where we fix $m=2, q_{1} \neq q_{2}, \theta_{0}=0, \theta_{2}=\theta_{1}+0.25$ and $\theta_{3}=1$. From this figure, we can see significant effects of the timing of pesticide applications on $x^{*}$, and the later the application of the pesticide the greater the reduction of the population size and consequently leads to avoidance of the paradox. Besides, effects of increasing the killing rates of different times of pesticide applications $\left(q_{1}\right.$ and $\left.q_{2}\right)$ affect each other significantly. Specifically, when the killing rate $q_{1}$ is large, increasing $q_{2}$ can greatly reduce the population size, while when $q_{1}$ is small, increasing $q_{2}$ can lead to an increase of the population size, as shown in Fig.5(a) and Fig.5(b).

In order to show the effects of the numbers of pesticide applications within each generation on the stable population size $x^{*}$, we fix the parameter values as those in Fig.6 and Fig.7 with $q_{k}=q(k=1, \cdots, m), \theta_{0}=0, \theta_{m+1}=1$, $\theta_{i+1}=\theta_{i}+\theta(i=1, \cdots, m)$, and then let both parameters $q$ and $\theta$ vary simultaneously. From Fig.6 we can see that for a relatively small killing rate (i.e. less than the threshold value $q_{c}$ ) and relatively small $\theta$, increasing the numbers of pesticide applications does not reduce the stable population size $x^{*}$ at all, no matter how many pesticide applications are made within each generation, which further confirms the main results shown in Theorem 1 and that the cumulative effects of incorrect use of pesticides may result in more severe pest outbreaks. However, the numbers of pesticide applications can contribute significantly to reducing the population size if the killing rate is large enough, as shown in Fig.6. Besides, the larger the number of pesticide applications is, the smaller is the value of threshold $q_{c}$. Moreover, it follows from Fig.7, we find that the stable population size $x^{*}$ at the threshold $q_{c}$ also decreases with the increase of the number and the timing of pesticide applications. Therefore, the number of pesticide applications, the timing of pesticide applications and the effectiveness of the pesticides are all crucial for pest control.

\subsection{Power function}

Based on Beverton-Holt and Ricker functions we have discussed the effects of the timing of pesticide applications and the killing rate on the stable 
population size in the above two subsections, but those effects cannot be investigated directly as the analytical formula of $x^{*}$ could not be obtained. Therefore, in the following we consider a special case which allows us to derive the analytical formula for $x^{*}$, i.e., the following power function [25]

$$
g(x)=x^{-\gamma}
$$

has been employed which depends on the effectiveness of the density, where the parameter $\gamma$ is a positive constant different from 1. Substituting (4.9) into model (2.4), yields the following model with multiple chemical controls within each generation

$$
x_{n+1}=\lambda \prod_{i=1}^{m}\left(1-q_{i}\right) x_{n}\left\{\sum_{i=0}^{m}\left[\Delta \theta_{i}\left(\prod_{j=0}^{i}\left(1-q_{j}\right) x_{n}\right)^{-\gamma}\right]\right\} .
$$

The equilibrium population size $x^{*}$ satisfies the following equation

$$
1=\lambda \prod_{i=1}^{m}\left(1-q_{i}\right)\left\{\sum_{i=0}^{m}\left[\Delta \theta_{i}\left(\prod_{j=0}^{i}\left(1-q_{j}\right) x^{*}\right)^{-\gamma}\right]\right\},
$$

which can be solved with respect to $x^{*}$, and we have

$$
x^{*}=\sqrt[\gamma]{\lambda \prod_{i=1}^{m}\left(1-q_{i}\right) \sum_{i=0}^{m}\left(\Delta \theta_{i}\left(\prod_{j=0}^{i}\left(1-q_{j}\right)^{-\gamma}\right)\right.} .
$$

Obviously $x^{*}$ is unique and positive under condition (3.2).?? For the stability of the equilibrium $x^{*}$, the function $f\left(x^{*}\right)$ becomes

$$
f\left(x^{*}\right)=\lambda \prod_{i=1}^{m}\left(1-q_{i}\right) x^{*}\left\{\sum_{i=0}^{m}\left[\Delta \theta_{i}\left(\prod_{j=0}^{i}\left(1-q_{j}\right) x^{*}\right)^{-\gamma}\right]\right\},
$$

and consequently we have

$$
f^{\prime}\left(x^{*}\right)=(1-\gamma) x^{*-\gamma} \lambda \prod_{i=1}^{m}\left(1-q_{j}\right)\left\{\sum_{i=0}^{m}\left[\Delta \theta_{i}\left(\prod_{j=0}^{i}\left(1-q_{j}\right)^{-\gamma}\right)\right]\right\} .
$$

Substituting Eq.(4.12) into Eq.(4.14), we get $f^{\prime}(x)=1-\gamma$ ? which means that if $0<\gamma<2(\gamma \neq 1)$ then we have $\left|f^{\prime}\left(x^{*}\right)\right|<1$. Thus the equilibrium 
of population size $x^{*}>0$ uniquely exists and is locally asymptotically stable provided that $0<\gamma<2(\gamma \neq 1)$.

It is easy to see that

$$
\frac{\partial x^{*}}{\partial q_{k}}=\frac{-F_{q_{k}}}{F_{x}}=0 \Rightarrow F_{q_{k}}=0,
$$

and denote $M=\lambda \prod_{i=1, i \neq k}^{m}\left(1-q_{i}\right)$, then we have

$$
\begin{aligned}
F_{q_{k}}= & M\left(1-q_{k}\right)\left\{\sum_{i=k}^{m} \Delta \theta_{i} \cdot \gamma\left(\prod_{j=0}^{i}\left(1-q_{j}\right) x^{*}\right)^{-\gamma-1}\left(\prod_{j=0, j \neq k}^{i}\left(1-q_{j}\right) x^{*}\right)\right\} \\
& -M\left\{\sum_{i=0}^{m}\left[\Delta \theta_{i}\left(\left(\prod_{j=0}^{i}\left(1-q_{j}\right) x^{*}\right)^{-\gamma}\right)\right]\right\} \\
= & M\left\{\sum_{i=k}^{m} \Delta \theta_{i} \cdot \gamma\left(\prod_{j=0}^{i}\left(1-q_{j}\right) x^{*}\right)^{-\gamma-1}\left(\prod_{j=0}^{i}\left(1-q_{j}\right) x^{*}\right)\right. \\
& \left.-\sum_{i=0}^{m}\left[\Delta \theta_{i}\left(\left(\prod_{j=0}^{i}\left(1-q_{j}\right) x^{*}\right)^{-\gamma}\right)\right]\right\} \\
= & M\left\{\sum_{i=k}^{m} \Delta \theta_{i} \cdot \gamma\left(\prod_{j=0}^{i}\left(1-q_{j}\right) x^{*}\right)^{-\gamma}-\sum_{i=0}^{m}\left[\Delta \theta_{i}\left(\left(\prod_{j=0}^{i}\left(1-q_{j}\right) x^{*}\right)^{-\gamma}\right)\right]\right\} \\
= & M\left\{\sum_{i=k}^{m} \Delta \theta_{i} \cdot(\gamma-1)\left(\prod_{j=0}^{i}\left(1-q_{j}\right) x^{*}\right)^{-\gamma}\right. \\
& \left.-\sum_{i=0}^{k-1}\left[\Delta \theta_{i}\left(\left(\prod_{j=0}^{i}\left(1-q_{j}\right) x^{*}\right)^{-\gamma}\right)\right]\right\} \\
= & 0 .
\end{aligned}
$$

Substituting Eq.(4.12) into $F_{q_{k}}$, then we can get that

$$
\begin{gathered}
\sum_{i=k}^{m} \frac{\Delta \theta_{i}(\gamma-1)\left(\prod_{j=0}^{i}\left(1-q_{j}\right)^{-\gamma}\right)}{\lambda \prod_{i=1}^{m}\left(1-q_{i}\right)\left[\sum_{i=0}^{m} \Delta \theta_{i}\left(\prod_{j=0}^{i}\left(1-q_{j}\right)\right)^{-\gamma}\right]} \\
-\sum_{i=0}^{k-1} \frac{\Delta \theta_{i}\left(\prod_{j=0}^{i}\left(1-q_{j}\right)^{-\gamma}\right)}{\lambda \prod_{i=1}^{m}\left(1-q_{i}\right)\left[\sum_{i=0}^{m} \Delta \theta_{i}\left(\prod_{j=0}^{i}\left(1-q_{j}\right)\right)^{-\gamma}\right]}=0 \\
\Rightarrow \quad \sum_{i=k}^{m} \Delta \theta_{i}(\gamma-1)\left(\prod_{j=0}^{i}\left(1-q_{j}\right)^{-\gamma}\right)-\sum_{i=0}^{k-1} \Delta \theta_{i}\left(\prod_{j=0}^{i}\left(1-q_{j}\right)^{-\gamma}\right)=0 \\
\Rightarrow \quad\left(1-q_{k}\right)^{-\gamma} \sum_{i=k}^{m} \Delta \theta_{i}(\gamma-1)\left(\prod_{j=0, j \neq k}^{i}\left(1-q_{j}\right)^{-\gamma}\right)=\sum_{i=0}^{k-1} \Delta \theta_{i}\left(\prod_{j=0}^{i}\left(1-q_{j}\right)^{-\gamma}\right) \\
\Rightarrow \quad\left(1-q_{k}\right)^{\gamma}=\frac{(\gamma-1) \sum_{i=k}^{m} \Delta \theta_{i}\left(\prod_{j=0, j \neq k}^{i}\left(1-q_{j}\right)^{-\gamma}\right)}{\sum_{i=0}^{k-1} \Delta \theta_{i}\left(\prod_{j=0}^{i}\left(1-q_{j}\right)^{-\gamma}\right)} .
\end{gathered}
$$

Therefore, these results confirm that there exists a threshold $q_{c}$ such that

$$
\frac{\partial x^{*}}{\partial q_{k}}=0 \Rightarrow q_{k}=q_{c}=1-\left[\frac{\left.(\gamma-1) \sum_{i=k}^{m} \Delta \theta_{i}\left(\prod_{j=0, j \neq k}^{i}\left(1-q_{j}\right)\right)^{-\gamma}\right)}{\sum_{i=0}^{k-1}\left(\Delta \theta_{i}\left(\prod_{j=0}^{i}\left(1-q_{j}\right)\right)^{-\gamma}\right)}\right]^{\frac{1}{\gamma}}
$$


and

$$
\frac{\partial x^{*}}{\partial q_{k}} \leq 0 \Rightarrow q_{k} \leq q_{c}, \quad \frac{\partial x^{*}}{\partial q_{k}}>0 \Rightarrow q_{k}>q_{c} .
$$

Based on the above discussion, we can conclude that

1. the paradox will occur if $0<\gamma<2(\gamma \neq 1)$ and $q>q_{c}$;

2. the paradox will not occur if one of the following conditions is admitted: $\gamma \geq$ 2 or $q \leq q_{c}$

This threshold condition $q>q_{c}$ can also be described as $\theta<\theta_{c}$. When $m=1, m=2, q_{k}=q(k=1)$ and $\theta_{i}+1=\theta_{i}+\theta$ the threshold $\theta_{c}$ can be expressed as follows:

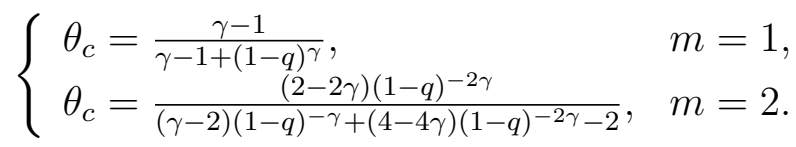

To see the parameter regions in which the paradox will occur clearly, we carried out numerical analysis, as described in the rest of this subsection. Fig. 8 shows the threshold values when $m=1$ and $m=2$ in which I represents the region where no paradox will occur and II represents the region in which the paradox will occur. From this figure we can see that large $\theta$ and small $q$ are conducive to avoiding the paradox.

According to Eq.(4.11) we can get the contour plot of the equilibrium population size under some specific parameter values as shown in Fig.9. Fig.9 indicates that the paradox can occur in all of the four circumstances. When the applications of pesticide are implemented early (as shown in Fig.9(a) and Fig.9(b)), increasing the killing rate $q_{1}$ or $q_{2}$ can only lead to an increase of the population size. However, when the interventions are implemented later (as shown in Fig.9(c) and Fig.9(d)), increasing the killing rate can result in a decrease at first and then an increase of the population size.

In order to show the comprehensive effects of the numbers of pesticide applications, the timing of pesticide applications and the killing rate on the stable population size $x^{*}$, we fix the parameter values as those in Fig.10 and Fig.11 with $\lambda=4, \gamma=1.3$ and $m=1,2,3,4$. It follows from Fig.10 and Fig.11 that the later the application of pesticide the greater the reduction of the population size and the increase of the value of the threshold $q_{c}$ which consequently helps avoidance of the paradox. Besides, we can see that increasing the number of pesticide applications can help to avoid the occurrence of the paradox(as shown in Fig.11(b)). Moreover, comparing Fig.11(a) 
with Fig.11(b) we find that the effects of increasing the number of pesticide applications varied with the timing of interventions. In particular, when $\theta$ is small, namely, implementing the pesticide application early, increasing the number of interventions can lead to an increase of the stable population size $x^{*}$, while when $\theta$ is relative large, increasing the number of interventions can lead to a decrease at first and then an increase of the stable population size $x^{*}$, which is opposite to the results of the Ricker model. Therefore, later and multiple pesticide applications will benefit pest control but a high killing rate is a disadvantage.

\section{Conclusions}

In real life, pesticides are usually applied several times by farmers once the first pesticide application has failed to maintain the pest population below a suitably low level. But sometimes things go against farmers wishes, and a more severe outbreak of the pest may occur. Therefore, in order to reveal this mechanism from a mathematical point of view, we have extended the discrete single species model with multiple instantaneous chemical controls within each pest generation, which allows us to analyse the side-effects of multiple pesticide applications on pest outbreaks, and consequently to improve the designs of successful pest control strategies.

The main results shown in Theorem 1 clarify the relations between the $k$-th application and the $(k+1)$-th application, and the relations between the $k$-th application and the $(k-1)$-th application. Under certain conditions, the occurrence of the paradox in the $k$-th application of pesticide indicates that the paradox must occur at the $(k-1)$-th application of pesticide. While if the $k$-th application of pesticide can successfully reduce the density of the pest population, then the same result can be obtained at the $(k+1)$-th application of pesticide. These interesting results indicate that the pesticide applications should be carefully designed before they are applied in practice, otherwise more severe outbreaks, in terms of the density of the pest population, will result.

The results also show that the earlier the pesticide application is, the more likely it is that the paradox will occur. Moreover, multiple pesticide applications could cause cumulative effects which result in more severe pest outbreaks if the pesticides have been used incorrectly. However, the numbers of pesticide applications can greatly contribute to reductions of the population size if the killing rate is large enough and the pesticides have been applied 
correctly, as shown in Fig.6. Thus, we conclude that the number of pesticide applications, the timing of pesticide applications and the effectiveness of the pesticides are the most important factors which affect the success or otherwise of pest control.

Compared with the results obtained in $[5,4,25]$, we see that the model with multiple pesticide applications within each pest generation will produce more interesting and complex results, and can help us to reveal how the multiple pesticide applications affect each other. Note that the present work mainly focused on the discrete single species model with multiple chemical controls, and IPM strategies based on the continuous modelling methods proposed in $[43,38,26,47]$ will be considered in the near future.

Acknowledgements This work was supported by the National Natural Science Foundation of China (NSFC 11471201), and by the Fundamental Research Funds for the Central Universities (GK201003001, GK201401004, GK201603003).

The authors declare that there is no conflict of interest regarding the publication of this paper 


\section{Reference}

[1] A.J. Lotka, Undamped oscillations derived from the law of mass action. J. Amer. Chem. Soc. 42 (1920) 1595-1599.

[2] V. Volterra, Variations and fluctuations of a number of individuals in animal species living together. Animal Ecology, Mcgraw Hill, New York, 1931.

[3] H. Seno, A paradox in discrete single species population dynamics with harvesting/thinning, Math. Biosci. 214 (2008) 63-69.

[4] Begoña Cid, Frank M. Hilker, Eduardo Liz, Harvest timing and its population dynamic consequences in a discrete single-species model. Math. Biosci. 248 (2014) 78-87.

[5] T. Matsuoka, H. Seno, Ecological balance in the native population dynamics may cause the paradox of pest control with harvesting. J. Theor. Biol. 252 (2008) 87-97.

[6] K, Shea. Management of populations in conservation, harvesting and control, Tree 13 (1998) 371.

[7] W.M. Getz, R.G. Haight, Population Harvesting: Demographic Models of Fish, Forest, and Animal Resources, Monographs in Population Biology. 27 (1989).

[8] C.W. Clark, Mathematical Bio-Economics: The Optimal Management of Renewable Resources, Wiley, New York, 1990.

[9] R.L. Metcalf, W.H. Luckmann, Introduction to Insect Pest Management, Wiley-Interscience, 1975.

[10] C.B. Huffaker, Theory and Practice of Biological Control, Academic Press, 1976.

[11] C.B. Huffaker, New Technology of Pest Control, Wiley-Interscience, 1980.

[12] R.E. Plant, Modeling and simulation in agricultural pest managenment, SIAM Rev, 29 (1987) 235-261. 
[13] W.W. Murdoch, C.J. Briggs, Theory for biological control: recent developments, Ecology, 77 (1996) 2001-2013.

[14] S.D. Lane, N.J. Mills, W.M. Getz, The effects of parasitoid fecundity and host taxon on the biological control of insect pests: the relationship between theory and data, Ecol.Entomol, 24 (1998) 181-190.

[15] M. Takagi, Perspective of practical biological control and population theories, Res.Popul.Ecol, 41 (1998) 121-126.

[16] M.E. Hochberg, R.I. Anthony, Parasitoid Population Biology, Princeton University Press, 2000.

[17] A.E. Hajek, M.L. McManus, I. Delalibera Jr, A review of introductions of pathogens and nematodes for classical biological control of insects and mites, Biol. Control, 41 (2007) 1-13.

[18] P. DeBach, D. Rosen, C.E. Keffett, Biological control of coccids by introduced natural enemies, Plenum Press, 1971.

[19] J.H. Perkins, Insects, Experts, and the Insecticide Crisis, Plenum Press, 1982.

[20] U. Gerson, E. Cohen, Resurgence of spider mites (Acari: Tetranychidae)induced by synthetic pyrethroids, Exp.Appl.Acarol, 6 (1989) 29-46,

[21] M.R. Hardin, B. Benrey, M. Coll, W.O. Lamp, G.K. Roderick, P. Barbosa, Arthropod pest resurgence: an overview of potential mechanisms, Crop. Prot. 14 (1995) 3-18.

[22] E. Cohen, Pesticide-mediated homeostatic modulation in arthropods, Pestic. Biochem. Physiol. 85 (2006) 21-27.

[23] J.G. Morse, Agricultural implications of pesticide-induced hormesis of insects and mites, Hum. Exp. Toxicol. 17 (1998) 266-269.

[24] D.G. James, T.S. Price, Fecundity in twospotted spider mite (Acari:Tetranychidae) is increased by direct and systemic exposure to imidacloprid, Ecotoxicology. 95 (2002) 729-732.

[25] Hiromi Seno, A paradox in discrete single species population dynamics with harvesting/thinning, Math. Biosci, 214 (2008) 63-69. 
[26] S.Y. Tang, W.H. Pang, R.A. Cheke, J.H. Wu, Global dynamics of a state-dependent feedback control system. Adv. Differ. Equ. 2015 2015:322. DOI 10.1186/s13662-015-0661-x

[27] J.G. Skellam, Random dispersal in theoretical populations, Bull. Math. Biol. 53 (1991) 135-165.

[28] R.J.H. Beverton, On the dynamics of exploited fish populations, Fishery Investigations, 1957.

[29] E.C. Pielou, An introduction to mathematical ecology, Wiley, 1969.

[30] B. Cid, F.M. Hilker, E. Liz, Harvest timing and its population dynamic consequences in a discrete single-species model, Math. Biosci. 248 (2013) 78-87.

[31] R.M. May, Stability and Complexity in Model Ecosystems, Princeton University Press, Princeton, NJ, 1974.

[32] P.A.P, Moran, Some remarks on animal population dynamics, Biometrics. 6 (1950) 250-258.

[33] W.E. Ricker, Stock and recruitment, J. Fish. Res. Bd. Can, 559 (1954) 11.

[34] L.M. Cook, Oscillation in the simple logistic growth model, Nature, 67 (1965) 316.

[35] R.M. May, Bioligical populations with non-overlapping generations: stable points, stable cycles, and chaos, Science, 186 (1974) 645.

[36] P. Collet, J.P. Eckmann, Iterated maps on the interval as dynamical systems, Birkhäusue, 1980.

[37] R. Seydel, Practical Bifurcation and Stability Analysis: From Equilibrium to Chaos, Springer, 1994.

[38] S.Y. Tang, Y.N. Xiao, L.S. Chen, Cheke RA, Integrated pest management models and their dynamical behaviour. Bull. Math. Biol. 67 (2005) 115-135. 
[39] J.C.V. Lenteren, Integrated pest management in protected crops, in: D.Dent (Ed.), Integrated pest management, Chapman \& Hall, London. (1995) 311-320.

[40] J.C.V. Lenteren, Measures of success in biological control of arthropods by augmentation of natural enemies, in: S.Wratten, G.Gurr(Eds.), Measures of Success in Biological Control, Kluwer Academic Publishers, Dordrecht. (2000) 77-89.

[41] S.Y. Tang, L.S. Chen, Multiple attractors in stage-structured population models with birth pulses. Bull. Math. Biol. 65 (2003) 479-495.

[42] S.Y. Tang, L.S. Chen, Modelling and analysis of integrated pest management strategy. Discrete. cont. dyn-B. 4 (2004) 759-768.

[43] S.Y. Tang, R.A. Cheke, Stage-dependent impulsive models of Integrated Pest Management (IPM) strategies and their dynamic consequences. J. Math. Biol. 50 (2005) 257-292.

[44] S.Y. Tang, G.Y. Tang, R.A. Cheke, Optimum timing for integrated pest management:Modelling rates of pesticide application and natural enemy releases. J. Theor. Biol. 264 (2010) 623-638.

[45] S.Y. Tang, Y.N. Xiao, R.A. Cheke, Multiple attractors of host-parasitoid models with integrated pest management strategies: Eradication, persistence and outbreak. Theor. Popul. Biol. 73 (2008) 181-197.

[46] S.Y. Tang, R.A. Cheke, Models for integrated pest control and their biological implications. Math. Biosci. 215 (2008) 115-125.

[47] S.Y. Tang, B. Tang, A.L. Wang, Y.N. Xiao, Holling II predator-prey impulsive semi-dynamic model with complex Poincare map. Nonlinear Dyn., 2015, DOI 10.1007/s11071-015-2092-3. 

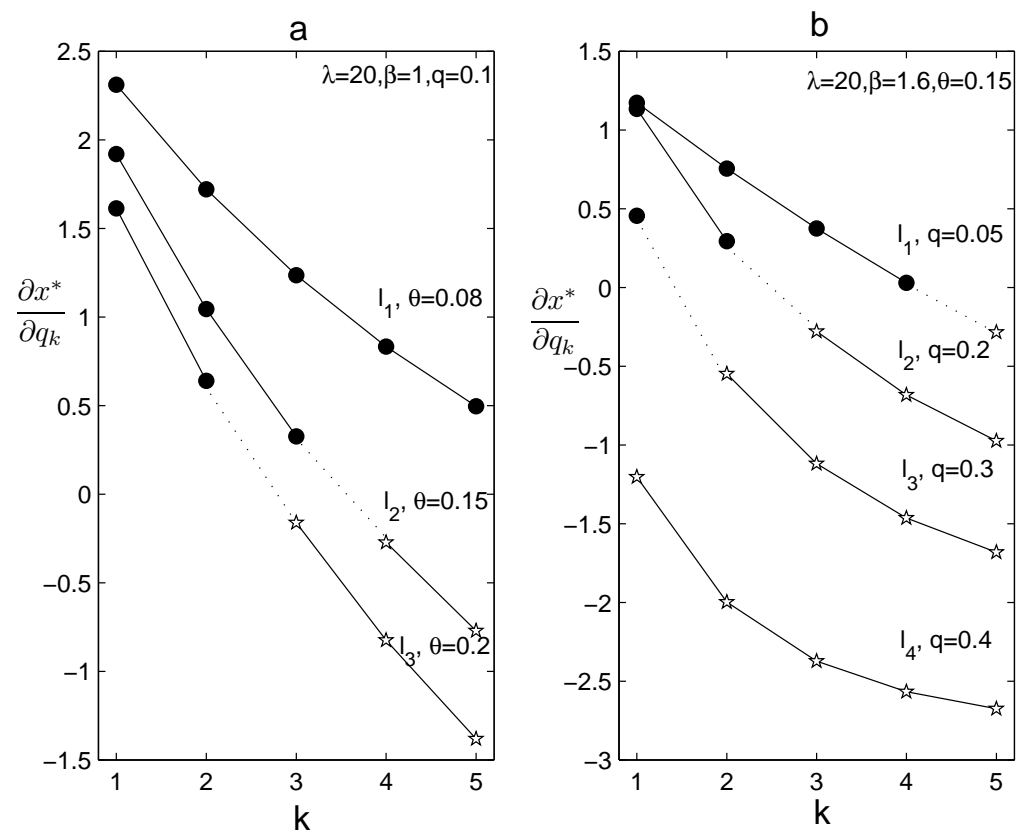

Figure 1: The illustrations of main results shown in Theorem 1.

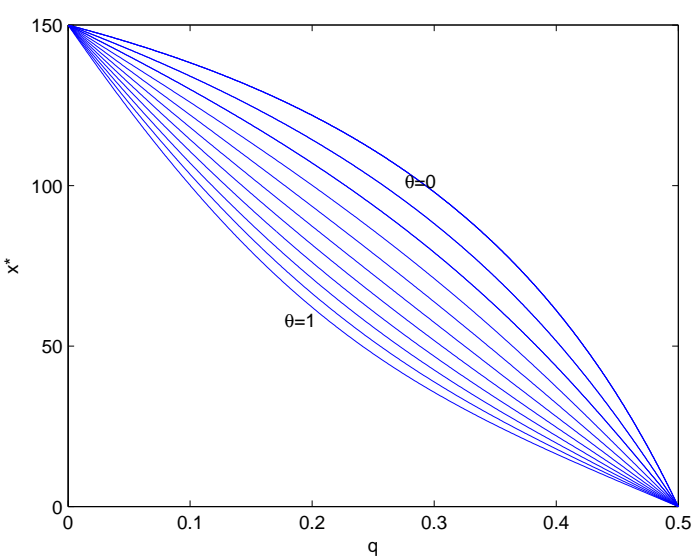

Figure 2: The equilibrium population size $x=x^{*}$ for model (4.2) as a function of killing rate $q$, with the variation of $\theta$ from 0 to 1 . The parameter values are fixed as follows: $\lambda=4.0, b=0.02, m=2, \theta_{0}=0, \Delta \theta_{i}=\theta_{i+1}-\theta_{i}(i=0,1,2), \theta_{3}=1$ and $q_{k}=q(k=1,2)$. 

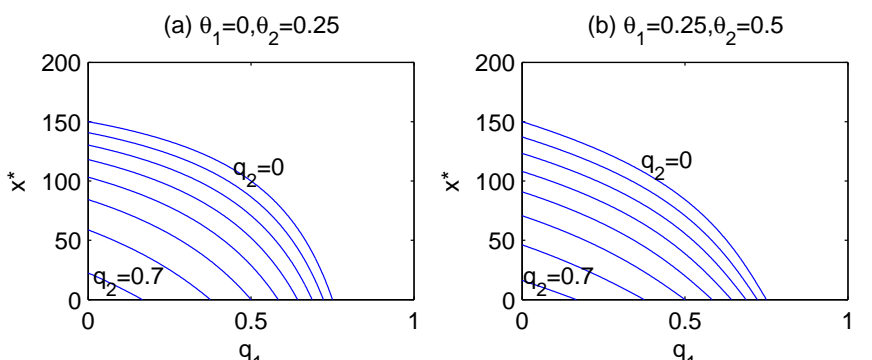

(c) $\theta_{1}=0.5, \theta_{2}=0.75$

(d) $\theta_{1}=0.75, \theta_{2}=1$
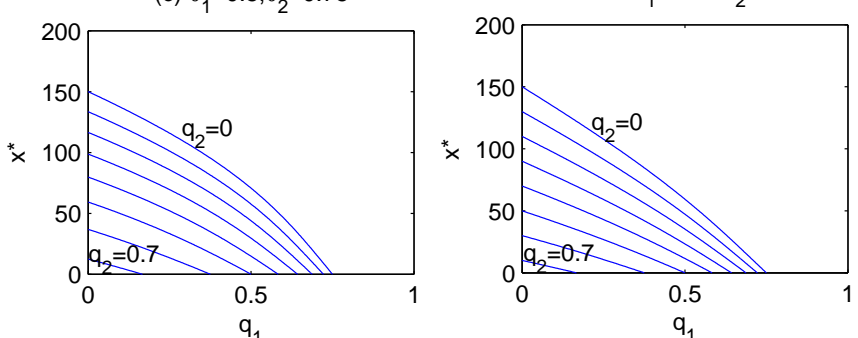

Figure 3: The equilibrium population size $x=x^{*}$ for model (4.2) as a function of killing rate $q$, with the variation of $q_{2}$ from 0 to 0.7 . The parameter values are fixed as follows: $\lambda=4.0, b=0.02, m=2, \theta_{0}=0 ; a: \theta_{1}=0$ and $\theta_{2}=0.25$; $b: \theta_{1}=0.25$ and $\theta_{2}=0.5 ; c: \theta_{1}=0.5$ and $\theta_{2}=0.75 ; d: \theta_{1}=0.75$ and $\theta_{2}=1$. 

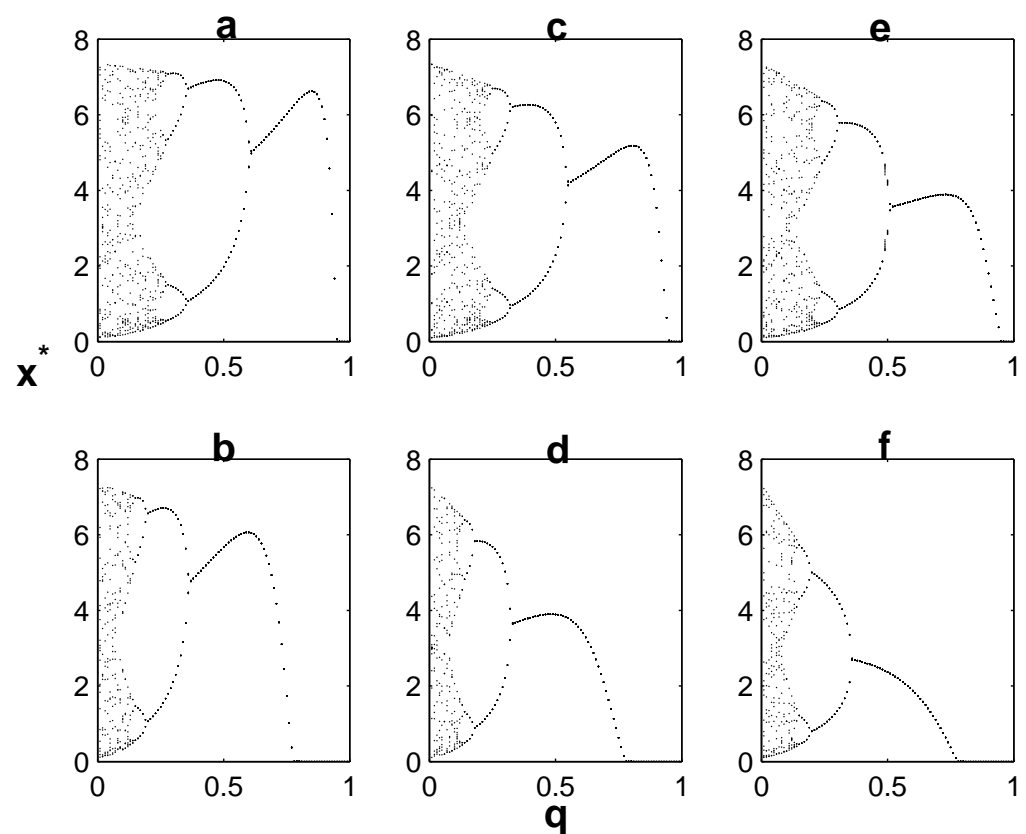

Figure 4: The effects of $\theta$ and $q$ on the stable equilibrium population level $x^{*}$ for model (4.5). The parameter values are fixed as follows: $\lambda=20, \beta=1.0$, and $m=1, \theta=0.1, q_{1}=q$ in a; $m=2, \theta=0.1, q_{1}=q_{2}=q$ in b; $m=1, \theta=0.3$ ? $q_{1}=q$ in $\mathrm{c} ; m=2, \theta=0.3, q_{1}=q_{2}=q$ in $\mathrm{d} ; m=1, \theta=0.5, q_{1}=q$ in e; $m=2$, $\theta=0.5, q_{1}=q_{2}=q$ in $\mathrm{f}$. 
(a) $\theta_{1}=0, \theta_{2}=0.25$

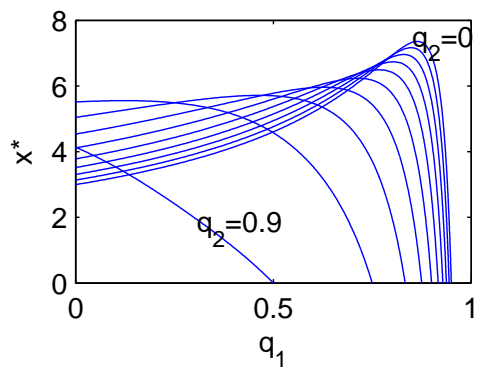

(c) $\theta_{1}=0.5, \theta_{2}=0.75$

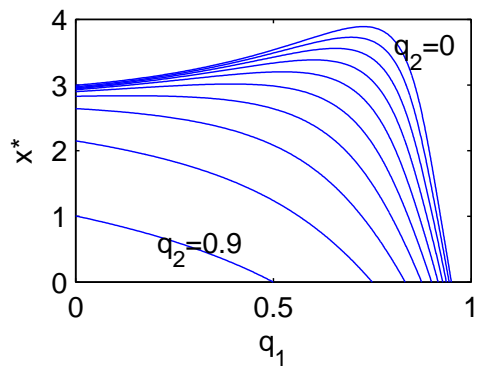

(b) $\theta_{1}=0.25, \theta_{2}=0.5$

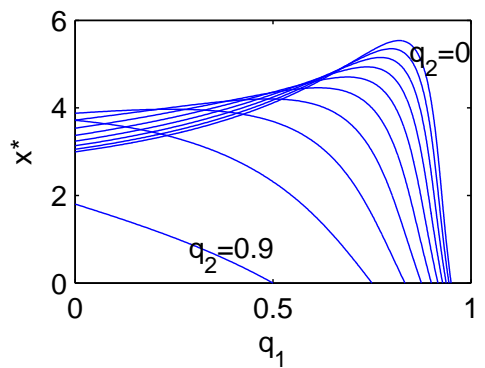

(d) $\theta_{1}=0.75, \theta_{2}=1$

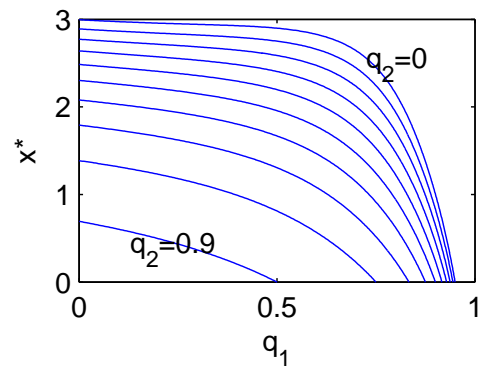

Figure 5: The equilibrium population size $x^{*}$ for model (4.5) as a function of $q_{1}$, with $q_{2}$ varying from 0 to 0.9 (with interval 0.1 ). The parameter values are fixed as follows: $\lambda=20, \beta=1.0, m=2, \theta_{0}=0, \theta_{3}=1$, and $\theta_{1}=0, \theta_{2}=0.25$ in a; $\theta_{1}=0.25, \theta_{2}=0.5$ in b; $\theta_{1}=0.5, \theta_{2}=0.75$ in c; $\theta_{1}=0.75, \theta_{2}=1$ in d. 
(a) $m=1$

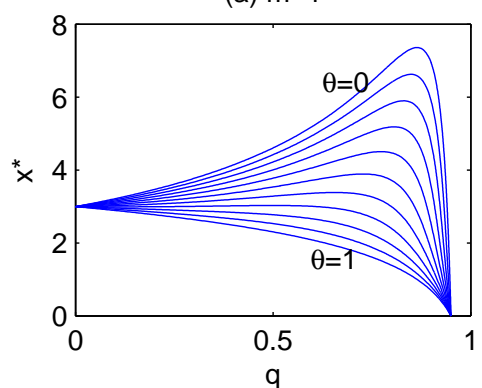

(c) $\mathrm{m}=3$

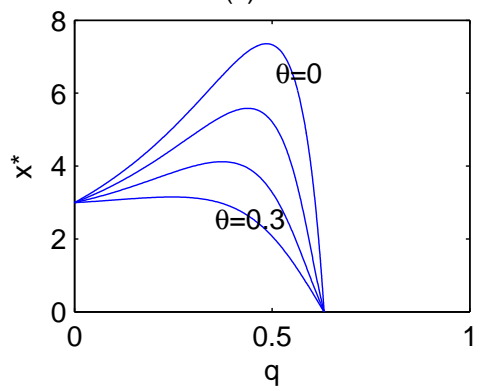

(b) $\mathrm{m}=2$

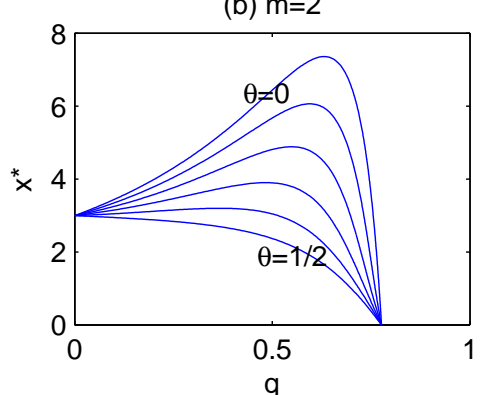

(d) $m=4$

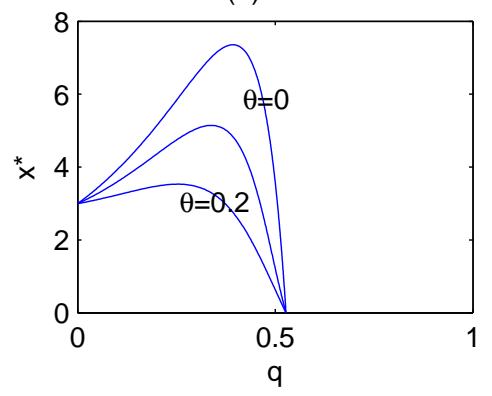

Figure 6: The equilibrium population size $x=x^{*}$ for model (4.5) as a function of killing rate $q$, with $\theta$ varying from 0 to $1 / \mathrm{m}$ (with interval 0.1 ). The parameter values are fixed as follows: $\lambda=20, \beta=1.0$ and $m=1,2,3,4$ in a, b, c, d, respectively. 
(a) $\theta=0.1$

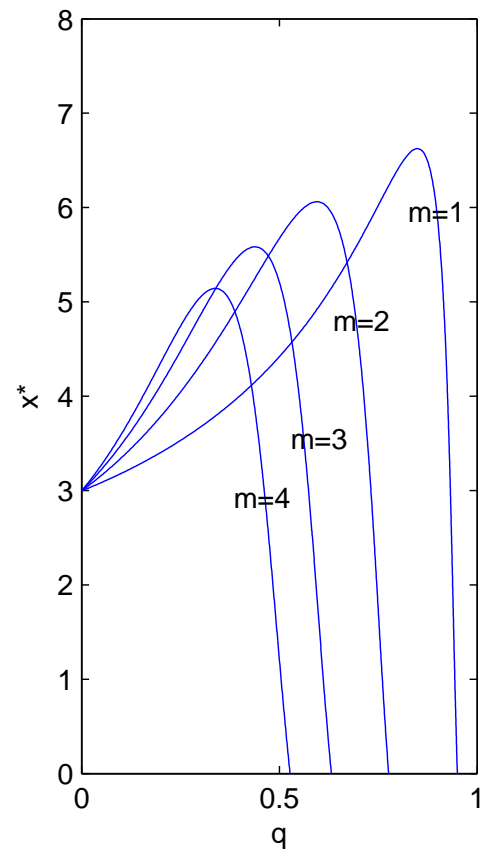

(b) $\theta=0.2$

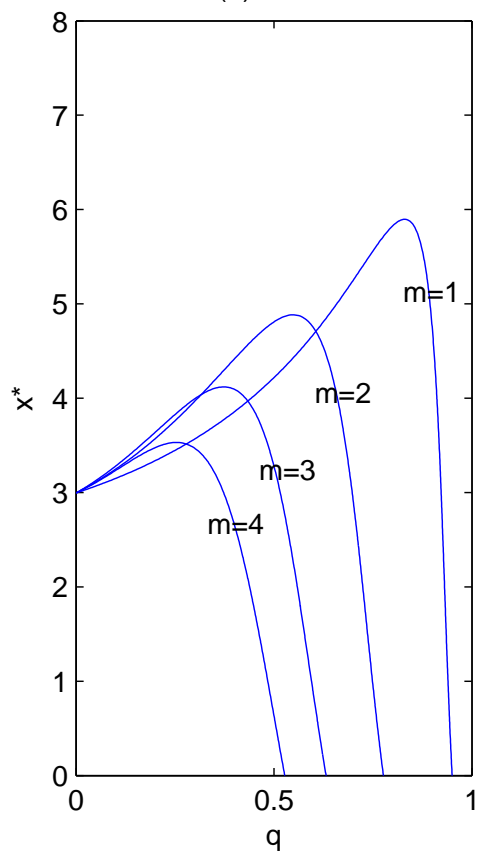

Figure 7: The equilibrium population size $x=x^{*}$ for model (4.5) as a function of $q$. The parameter values are fixed as follows: $\lambda=20, \beta=1.0, \theta=0.1,0.2$ in $\mathrm{a}, \mathrm{b}$ respectively and $m=1,2,3,4$ in each subfigure respectively. 
(a)

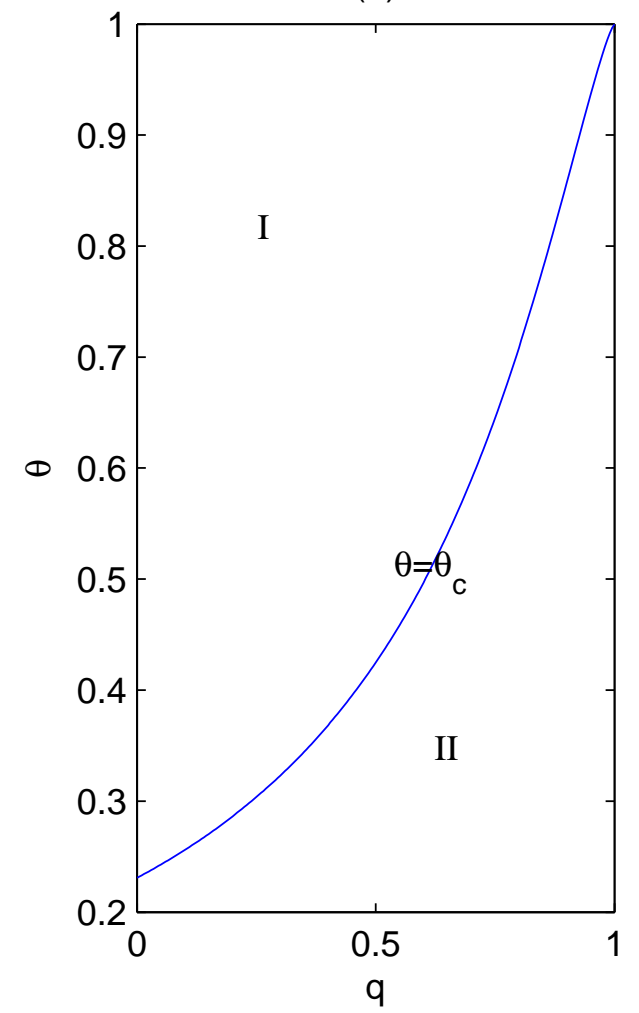

(b)

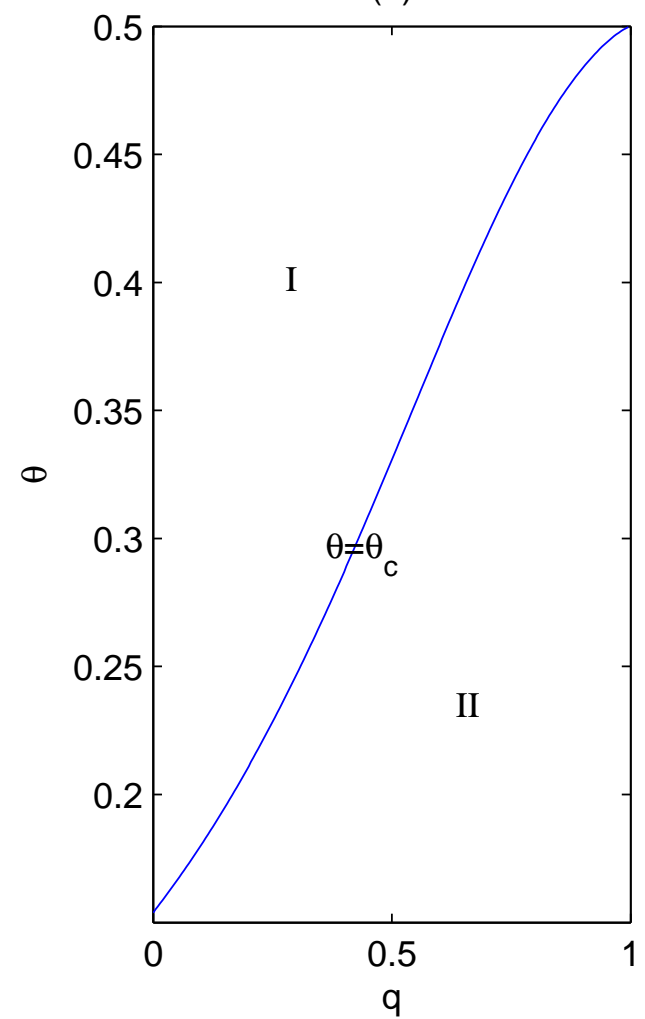

Figure 8: $(\gamma, \theta)$ and $(q, \theta)$-dependence of the emergence of paradox for the Power function model (4.10). The parameter values are fixed as follows: $\lambda=4.0, \gamma=1.3$, I: No paradox, II: Paradox, $a: m=1$ and $q_{1}=q ; b: m=2, q_{1}=q_{2}=q, \theta_{1}=\theta$, $\theta_{2}=\theta_{1}+\theta$. 
(a) $\theta_{1}=0, \theta_{2}=0.25$

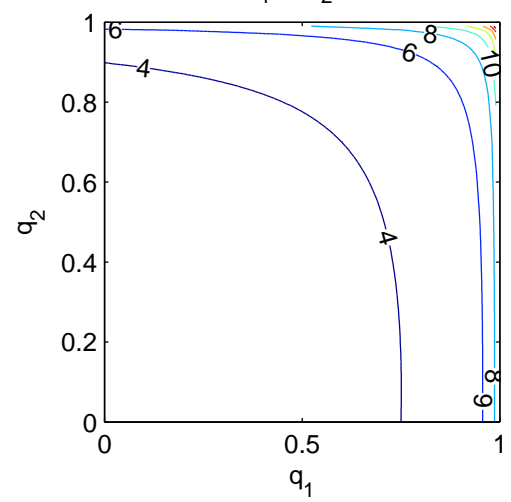

(c) $\theta_{1}=0.5, \theta_{2}=0.75$

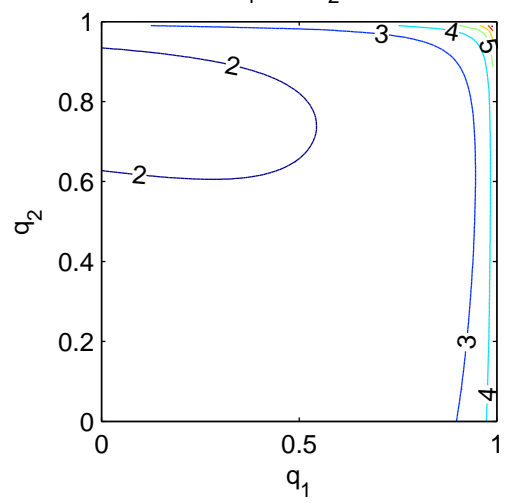

(b) $\theta_{1}=0.25, \theta_{2}=0.5$

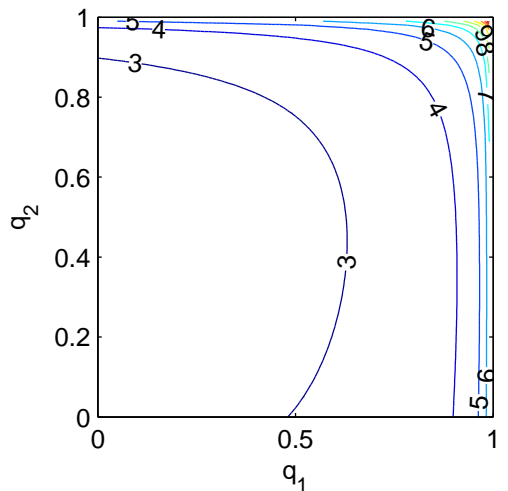

(d) $\theta_{1}=0.75, \theta_{2}=1$

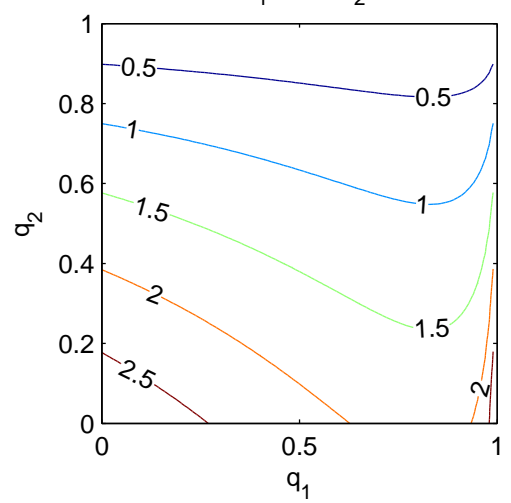

Figure 9: Contour plot of the equilibrium population size $x^{*}$ for model (4.10) with respect to $q_{1}$ and $q_{2}$. The parameter values are fixed as follows: $\lambda=4, \gamma=1.3$, $m=2, \theta_{0}=0, \theta_{3}=1$, and $\theta_{1}=0, \theta_{2}=0.25$ in a; $\theta_{1}=0.25, \theta_{2}=0.5$ in b; $\theta_{1}=0.5, \theta_{2}=0.75$ in $\mathrm{c} ; \theta_{1}=0.75, \theta_{2}=1$ in $\mathrm{d}$. 
(a) $m=1$

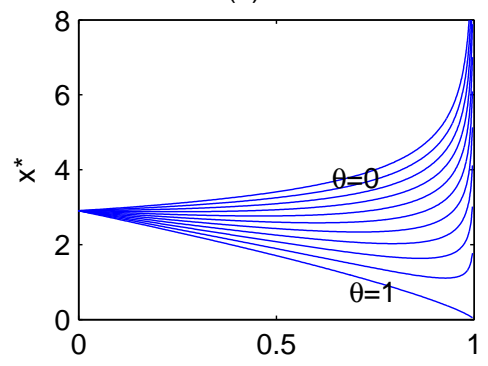

(c) $\stackrel{q}{m}=3$

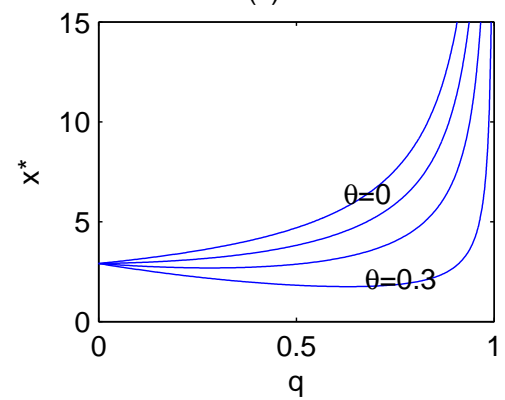

(b) $m=2$

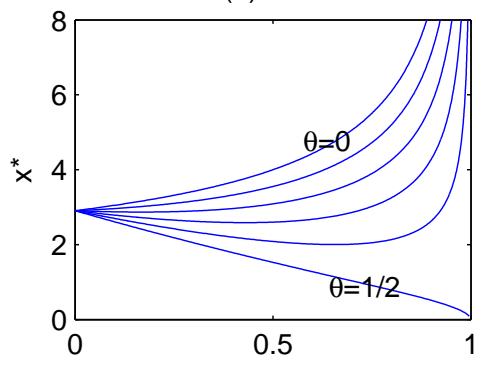

(d) $\stackrel{q}{m=4}$

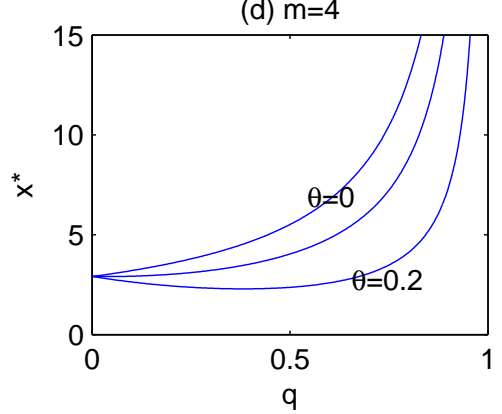

Figure 10: The equilibrium population size $x=x^{*}$ for model (4.10) as a function of killing rate $q$, with $\theta$ varying from 0 to $1 / \mathrm{m}$ (with interval 0.1 ). The parameter values are fixed as follows: $\lambda=4, \gamma=1.3$ and $m=1,2,3,4$ in a, b, c, d, respectively. 

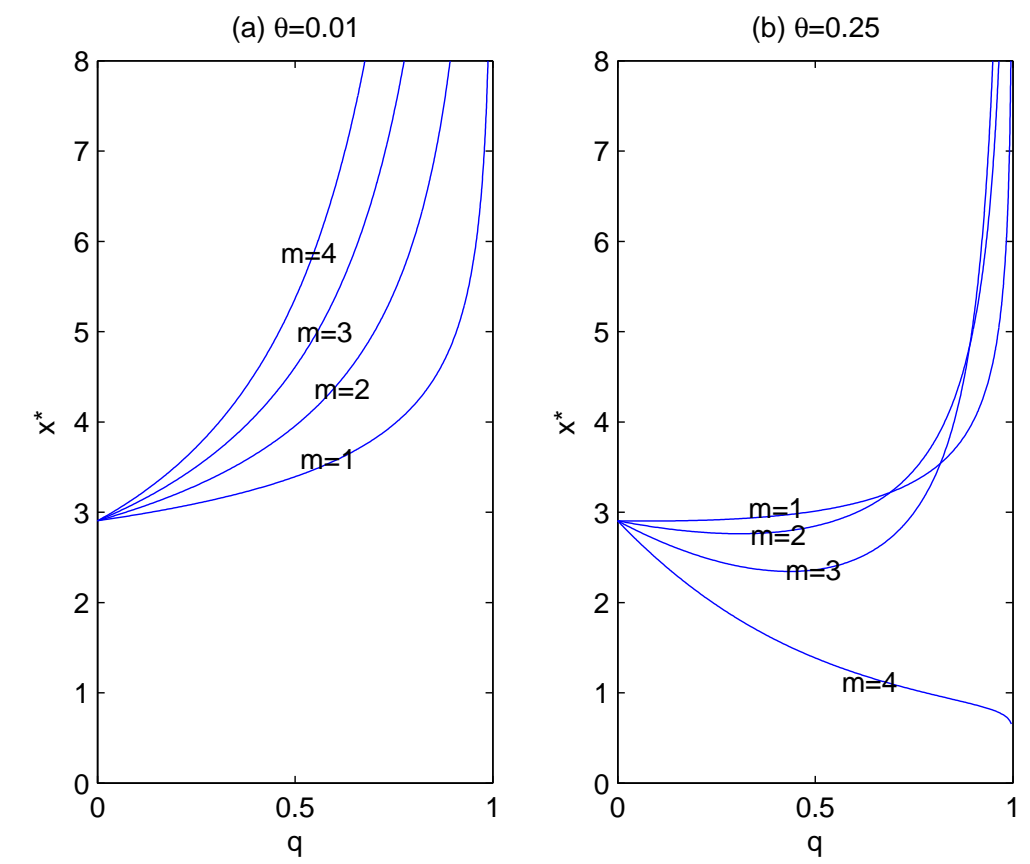

Figure 11: The equilibrium population size $x=x^{*}$ for model (4.10) as a function of killing rate $q$. The parameter values are fixed as follows: $\lambda=4, \gamma=1.3$ $\theta=0.01,0.25$ in a, b, respectively and $m=1,2,3,4$ in each subplot respectively. 